\title{
Simulation of the interstellar scintillation and the extreme scattering events of pulsars
}

\author{
M. Hamidouche ${ }^{1,2}$ and J.-F. Lestrade ${ }^{3}$ \\ 1 LPCE/CNRS, 3A Av. de la Recherche Scientifique, 45071 Orléans, France \\ 2 Astronomy Department, University of Illinois at Urbana-Champaign, 1002 W Green St, Urbana, IL 61801, USA \\ e-mail: mhamidou@astro.uiuc . edu \\ ${ }^{3}$ LERMA/CNRS, Observatoire de Paris, 77 Av. Denfert Rochereau, 75014 Paris, France \\ e-mail: jean-francois.lestrade@obspm.fr
}

Received 13 May 2004 / Accepted 10 March 2007

\begin{abstract}
The rare and conspicuous flux density variations of some radio sources (extragalactic and pulsars) for periods of weeks to months have been denoted Extreme Scattering Events (ESE's) by Fiedler et al. (1987, Nature, 362, 675). Presently, there is no astrophysical mechanism that satisfactorily produces this phenomenon. In this paper, we conjecture that inhomogeneities of the electronic density in the turbulent interstellar medium might be the origin of this phenomenon. We have tested this conjecture by a simulation of the scintillation of the pulsar B1937+21 at $1.4 \mathrm{GHz}$ and $1.7 \mathrm{GHz}$ for a period of six months. To this end, we have constructed a large square Kolmogorov phase screen made of $131 \mathrm{k} \times 131 \mathrm{k}$ pixels with electron inhomogeneity scales ranging from $6 \times 10^{6} \mathrm{~m}$ to $10^{12} \mathrm{~m}$ and used the Kirchhoff-Fresnel integral to simulate dynamic spectra of a pulsar within the framework of Physical Optics.

The simulated light curves exhibit a 10 day long variation simultaneously at 1.41 and $1.7 \mathrm{GHz}$ that is alike the "ESE" observed with the Nançay radiotelescope toward the pulsar B1937+21 in October 1989. Thus, we conclude that the "ESE" toward pulsars can be caused naturally by the turbulence in the ionized interstellar medium. This is instead of discrete over pressured ionized clouds crossing the line of sight as in the model of Fiedler et al. (1987, Nature, 362, 675). We suggest that longer events could occur in a simulation of scintillation, if larger electron inhomogeneities $\left(>10^{12} \mathrm{~m}\right)$ were included in the construction of the Kolmogorov phase screen. This next step requires a supercomputer.
\end{abstract}

Key words. scattering - turbulence - ISM: structure - stars: pulsars: general

\section{Introduction}

The rare and conspicuous flux density variations of some radio sources for periods of weeks to months have been denoted Extreme Scattering Events (ESE's). Usually, the flux density drops by at least $50 \%$ and increases somewhat at ingress and egress although there is a variety of possible shapes. The first event was recognized in the direction of the quasar $0954+658$ at 2.7 and $8.4 \mathrm{GHz}$ (Fiedler et al. 1987). The first event associated with a pulsar was detected in the direction of the millisecond pulsar B1937+21 at $1.41 \mathrm{GHz}$ (Cognard et al. 1993). The most recent census indicates that 15 ESE's have been identified in the radio light curves of 12 quasars in the entire Green Bank Interferometer monitoring of 149 radio sources at $\mathrm{GHz}$ frequencies between 1979 and 1996 (Lazio et al. 2001). Five ESE's have been identified in the direction of the pulsar B1937+21 between 1989 and 1996 (Lestrade et al. 1998, LRC98 hereafter) and a very long event was observed in the direction of the pulsar J1643-1224 (Maitia et al. 2003). Hill et al. (2005) also report criss-cross pattern in the dynamic spectra of PSR B0834+06 at $327 \mathrm{MHz}$ they interpret as resulting from compact scattering objects essentially stationary in the screen in 20 years.

The mechanism usually invoked for this phenomenon is scattering by a plasma lens that occurs when a discrete ionized cloud crosses the line of sight. Fiedler et al. (1987) proposed that the medium inside the cloud is highly turbulent, causing extreme scattering responsible for the flux density variation and source broadening. Differently, Romani et al. (1987) proposed that the medium inside the cloud acts as a purely refractive lens producing extreme refraction with caustics responsible for the flux variation and source displacement during the event. The observations of ESE's in directions of quasars and pulsars and these models provide an estimation of the cloud size of 1-50 AU and internal electronic density of $100-1000 \mathrm{e}^{-} \mathrm{cm}^{-3}$ (Fiedler et al. 1994). Therefore, such a structure is over pressurized relative to the ambient medium making its lifetime as short as a few years. In addition, the observed rate of occurrence implies a cloud space density as high as $\sim 10^{6}$ clouds $\mathrm{pc}^{-3}$ (LRC98). There is no known mechanism that can produce so many discrete ionized clouds in the ISM. In order to avoid this difficulty, we conjecture in this paper that the Kolmogorov turbulence of the ionized ISM is the natural cause for the ESE's and their occurrence is statistical.

In order to support this proposition, we have simulated interstellar scintillation at 1.41 and $1.7 \mathrm{GHz}$ toward the pulsar B1937+21 to compare to the observations taken at the Nançay radiotelescope ${ }^{1}$. The flux density of this pulsar measured at the Nançay radiotelescope is an average over an integration time of $70 \mathrm{~min}$ and an integration bandwidth of $7.5 \mathrm{MHz}$ centered around $1.41 \mathrm{GHz}$ and $12.5 \mathrm{MHz}$ around $1.7 \mathrm{GHz}$. These intervals are large enough to average several diffractive interstellar scintillation patterns in the time-frequency domain, and so essentially sample the refractive regime. However, some significant

\footnotetext{
Nançay radiotelescope is an instrument of the Observatoire de Nançay, France.
} 
influence of diffractive ISS remains. Therefore, we conducted a full scintillation calculation including the diffractive and refractive scales. The refractive time scales measured at Nançay in the direction of $\mathrm{B} 1937+21$ are $16 \pm 1$ days and $8 \pm 1$ days, at $1.41 \mathrm{GHz}$ and $1.7 \mathrm{GHz}$ respectively. Also, the modulation indexes are $m_{\mathrm{r}}=0.30 \pm 0.02$ and $0.50 \pm 0.04$ at $1.41 \mathrm{GHz}$ and $1.7 \mathrm{GHz}$ respectively. The zero-lag correlation of the flux density between these two frequencies is $0.93 \pm 0.05$. Detailed descriptions of these measurements are given in LRC98.

For the simulation presented in this paper, we have used the Kolmogorov 3D power spectrum of the electronic density $\delta n_{\mathrm{e}}(x, y, z)$ in the interstellar medium as established locally $(<1 \mathrm{kpc})$ by Armstrong et al. (1995). We have used the Kirchhoff-Fresnel integral to compute pulsar dynamic spectra in the framework of Physical Optics. In Sect. 2, we present the formulae for this simulation carried out in the thin screen approximation, the construction of the Kolmogorov phase screen and the computation of the diffraction pattern (see also Hamidouche 2003). In Sect. 3 (see also Appendix A), we construct a large square Kolmogorov phase screen of $131 \mathrm{k} \times 131 \mathrm{k}$ pixels. In Sect. 4 and in Appendix B, we make a series of tests to empirically determine how to set the parameters of the Kirchoff-Fresnel integral. In Sect. 5, we present the result of the simulation in the direction of $\mathrm{B} 1937+21$ simulated over a period of 6 months.

\section{Scattering of a point-like source by the ionized interstellar medium}

The historical problem of diffraction have been cast in modern mathematical terms by Born \& Wolf (1999) and Goodman (1968). A particularly clear presentation of this problem applied to interstellar optics in the radio domain is in Narayan (1992) and Gwinn et al. (1998). The tenuous interstellar plasma is a random medium where inhomogeneities of the density of free electrons produce fluctuations of the index of refraction. The radio wavefronts emitted by a point-like source (pulsar) and propagating through such a medium produce rapid intensity fluctuations at the Earth. These fluctuations are due to diffraction speckles in the plane of the observer with a short time scale of several minutes and over a narrow bandwidth $(\sim 1 \mathrm{MHz})$ at radio frequencies. This is the DISS phenomenon (Diffractive interstellar medium scintillation). This random medium produces also slow intensity variations of $10-100 \%$ over several days or more and over a broad bandwidth (>100 MHz). This is the RISS phenomenon (Refractive interstellar medium scintillation). Rickett (1988) proposes a concise presentation of these phenomena and of their main observables. Rickett (1977), Blandford \& Narayan (1985) and Rickett (1990) provide a complete derivation of these observables as functions of the random medium physical properties. Diffractive scintillation in dynamic spectra of pulsars is caused by medium inhomogeneities, whose scale $s_{0}$ is called diffractive scale or coherence length. From observations of pulsars at frequency around $1 \mathrm{GHz}$, this diffractive scale is between $10^{5} \mathrm{~m}$ and $10^{8} \mathrm{~m}$. Refractive scintillation of pulsars depends on medium inhomogeneities whose scales are about the scattering disk radius $r_{\mathrm{S}}=\theta_{\mathrm{S}} L$ where $L$ is the distance to the equivalent turbulent screen and $\theta_{\mathrm{S}}=\frac{\lambda}{2 \pi s_{0}}$ is the scattering angle. From pulsar observations, this refractive scale $r_{\mathrm{S}}$ is between $10^{10} \mathrm{~m}$ and $10^{13} \mathrm{~m}$. These two main scales of the ionized interstellar medium are suggestively sketched in Cordes et al. (1986). The line of sight to a pulsar moves through the medium with a significant transverse velocity $V_{\perp}$ and continuously samples inhomogeneities of sizes $s_{0}$ and $r_{\mathrm{S}}$ so that the diffractive timescale is $t_{\mathrm{d}}=s_{0} / V_{\perp}$ and the refractive timescale is $t_{\mathrm{r}}=L \theta_{\mathrm{S}} / V_{\perp}$. The decorrelation bandwidth $\Delta v_{\mathrm{dc}}=1 / 2 \pi \tau_{\mathrm{S}}$ observed in dynamic spectra of pulsars is associated with the temporal broadening of pulsars $\tau_{\mathrm{S}} \sim L \theta_{\mathrm{S}}^{2} / 2 c$ (Rickett 1988). The refractive modulation index $\left(m_{\mathrm{r}}=\mathrm{rms}\right.$ of intensity / mean intensity) is an important quantity directly comparable to observations and is theoretically $m_{\mathrm{r}}=1.08\left(\Delta v_{\mathrm{d}} / v\right)^{1 / 6}$ (Appendix B in Gupta et al. 1993). Pulsar dynamic spectra provide measurements of the diffractive parameters $t_{\mathrm{d}}$ and $\Delta v_{\mathrm{dc}}$ that yield the turbulence strength $C_{n}^{2}$ (e.g. Gupta et al. 1994). Long term pulsar flux density series yields the refractive parameter $t_{\mathrm{r}}$ that is typically in tens of days and the modulation index $m_{\mathrm{r}}<1$ (Stinebring et al. 2000).

Armstrong et al. (1995) analyze a large corpus of radioastronomy observations to establish that the 3D space power spectrum $P_{3 N}\left(q_{x}, q_{y}, q_{z}\right)$ of the free electron density $\delta n_{\mathrm{e}}(x, y, z)$ in the local interstellar medium $(<1 \mathrm{kpc})$ is of the Kolmogorov turbulence type. They give evidence of the power spectrum $P_{3 N}\left(q_{x}, q_{y}, q_{z}\right)=C_{n}^{2} \times\left(\sqrt{q_{x}^{2}+q_{y}^{2}+q_{z}^{2}}\right)^{-11 / 3}$ valid over 6 decades, $10^{-12} \mathrm{~m}^{-1}<\sqrt{q_{x}^{2}+q_{y}^{2}+q_{z}^{2}}<10^{-6} \mathrm{~m}^{-1}$. They suggest that it might extend over 12 decades. The derived turbulence strength by the same authors is $C_{n}^{2} \sim 10^{-3} \mathrm{~m}^{-20 / 3}$. This value can be considered as typical but large deviations are found depending on the celestial direction $10^{-4}<C_{n}^{2}<10^{-1} \mathrm{~m}^{-20 / 3}$ (Johnston et al. 1998; Cordes et al. 1985).

The duration of ESE's (weeks to years; Maitia et al. 2003) and the typical transverse velocity of pulsar (tens of $\mathrm{km} \mathrm{s}^{-1}$; Gupta et al. 1994) would make these events associated with large-scale inhomogeneities of free electrons, i.e. the refractive scales $r_{\mathrm{S}}\left(10^{10} \mathrm{~m}\right.$ and $\left.10^{13} \mathrm{~m}\right)$ following our conjecture. To test this concept, we simulate the scintillation of the pulsar $\mathrm{B} 1937+21$ at 1.41 and $1.7 \mathrm{GHz}$ with a Kolmogorov phase screen as large as $10^{12} \mathrm{~m} \times 10^{12} \mathrm{~m}$ sampled by $131 \mathrm{k} \times 131 \mathrm{k}$ pixels. The $3 \mathrm{D}$ nature of this simulation is reduced advantageously to a $2 \mathrm{D}$ problem in the thin screen approximation valid in the interplanetary and interstellar tenuous plasma. Following Salpeter (1967) and Lovelace (1970), this approximation is the limit where the ray lateral deflection is weak relative to the radial phase fluctuations. In this condition, the phase of a wavefront reduces to $\phi(x, y)=\int_{0}^{z} \lambda r_{\mathrm{e}} \delta n_{\mathrm{e}}(x, y, z) \mathrm{d} z$, with the electron radius $r_{\mathrm{e}}=2.8179 \times 10^{-13} \mathrm{~cm}$. The propagation of a wavefront from a source to an observer can be computed by the Kirchhoff-Fresnel diffraction integral (Born \& Wolf 1999). This integral relates the electrical field $\boldsymbol{E}\left(\boldsymbol{x}^{\prime}\right)$ in the observer's plane to the field $\boldsymbol{E}_{\mathrm{s}}$ emitted by a distant point-like source. Classically, this integral yields the diffraction pattern of an unperturbed aperture. We modify this integral by adding the Kolmogorov phase field $\phi_{K}(\boldsymbol{x})$ of a turbulent equivalent screen to model the wavefront corrugations caused by the ionized ISM. The electric field in the observer's plane with the Kirchhoff-Fresnel integral is:

$\boldsymbol{E}\left(\boldsymbol{x}^{\prime}\right)=\frac{\boldsymbol{E}_{\mathrm{s}}}{\lambda|\boldsymbol{L}|} \int_{\mathcal{S}} \mathrm{e}^{\mathrm{i}\left[\frac{2 \pi}{\lambda}|\boldsymbol{\ell}|+\phi_{K}(\boldsymbol{x})\right]} \mathrm{d} S$

where $\lambda$ is the wavelength, $\boldsymbol{x}^{\prime}$ is the observer position vector in a plane parallel to the sky plane, $\boldsymbol{x}$ is the position vector of a point in the phase screen plane $\mathcal{S}$ also parallel to the sky plane, $\boldsymbol{L}$ is the vector between the observer and the screen planes. In addition, $\left|\boldsymbol{x}^{\prime}\right|$ and $|\boldsymbol{x}|$ are very small with respect to $|\boldsymbol{L}|$ in our application below. This explains why the factor $\frac{1}{\lambda|\boldsymbol{L}|}$ is constant in Eq. (1). $|\boldsymbol{\ell}|$ is the full geometric path from the pulsar to the observer through the screen. The geometry is sketched in Fig. 1.

The 2D power spectrum of this phase screen $P_{2 \phi}\left(q_{x}, q_{y}\right)$ is related to the $3 \mathrm{D}$ Kolmogorov power spectrum of the 


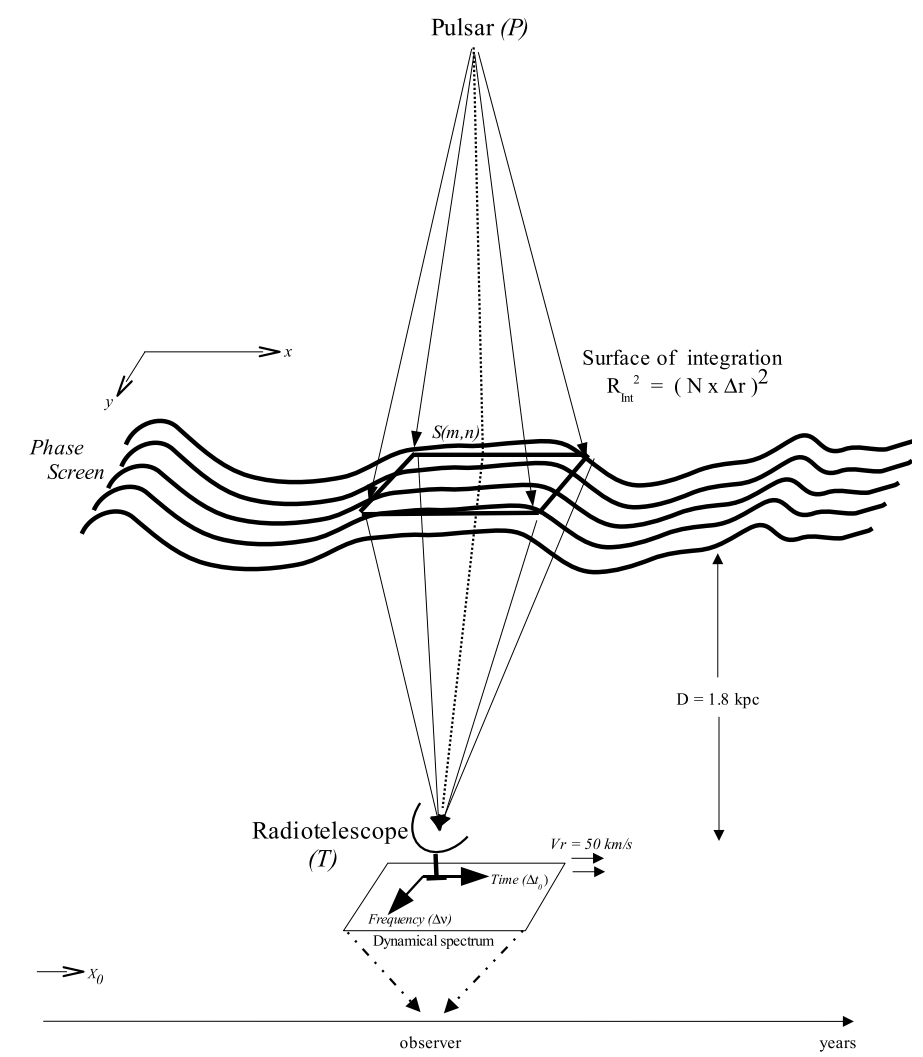

Fig. 1. Sketch of our computation of the dynamic spectrum of a pulsar seen through a $2 \mathrm{D}$ turbulent equivalent phase screen. The geometric path $\ell$ in the Kirchhoff-Fresnel integral, Eq. (1), is from the point source pulsar $P$ to the radiotelescope position $T$, by crossing the phase screen at the running pixel $S(m, n)$.

interstellar electronic density $P_{3 N}\left(q_{x}, q_{y}, q_{z}=0\right)$ as shown in Lovelace (1970) and Lovelace et al. (1970) in the thin screen approximation:

$P_{2 \phi}\left(q_{x}, q_{y}\right)=2 \pi z\left(\lambda r_{\mathrm{e}}\right)^{2} P_{3 \mathrm{~N}}\left(q_{x}, q_{y}, q_{z}=0\right)$

$z$ is the screen thickness. The thin screen approximation refers to a small deviation in the propagation direction of the wave rather than to a small thickness (Salpeter 1967).

In Appendix A, we derive the expression of the phase field $\phi_{K}(x, y)$ by using this relationship and the definition of the $2 \mathrm{D}$ average power spectrum $P_{2 \phi}\left(q_{x}, q_{y}\right)$ over the square screen surface $N^{2} \Delta r^{2}$ recalled here as:

$P_{2 \phi}\left(q_{x}, q_{y}\right)=\frac{\left|\mathcal{F}_{2 \phi}\left(q_{x}, q_{y}\right)\right|^{2}}{N^{2} \Delta r^{2}}$

$\mathcal{F}_{2 \phi}\left(q_{x}, q_{y}\right)$ is the Fourier Transform of the phase field $\phi_{K}(x, y)$. In Appendix A, $\phi_{K}(x, y)$ is cast into a discrete form for computation by FFT. The observer's plane $\boldsymbol{x}=(x, y)$ becomes a grid $(m, n)$ such as $x=m \Delta r$ and $y=n \Delta r$ with the grid step $\Delta r$. The spatial frequency plane $\left(q_{x}, q_{y}\right)$ becomes the grid $(k, l)$ such as $q_{x}=k \mathrm{~d} q_{x}$ and $q_{y}=l \mathrm{~d} q_{y}$. The frequency step $\mathrm{d} q_{x}$ is chosen to be the lowest spatial frequency $q_{\min , x}=1 / N \Delta r$ along $x$ and, similarly, $\mathrm{d} q_{y}=1 / N \Delta r$ along $y$. The grid step $\Delta r$ is chosen to be $s_{0} / 4$ after the tests described in Appendix B and adopting the notation $s_{0}$ for the coherence length. The maximum spatial frequency $q_{\max }=\frac{1}{2 \Delta r}$ in the integral (A.5) satisfies the Nyquist sampling rate. The discrete formula reduced to the case of the square screen is:

$$
\begin{aligned}
\phi_{K}(m, n)= & 2 \pi^{\frac{(1-\beta)}{2}} b \lambda r_{\mathrm{e}} \sqrt{z C_{N}^{2}}(N \Delta r)^{-1+\beta / 2} \\
& \times \sum_{k=-N / 2}^{k=+N / 2} \sum_{l=-N / 2}^{l=+N / 2}\left(k^{2}+l^{2}\right)^{-\beta / 4} \mathrm{e}^{-\frac{2 \pi \mathrm{i}}{N}(k m+l n)} \mathrm{e}^{-\mathrm{i} \psi(k, l)}
\end{aligned}
$$

The grid step $\Delta r$ conveniently appears as part of the multiplying factor in Eq. (4) so that the double summation of this equation can be computed once and stored in a file for use with different values of $\Delta r$. This was particularly useful for the tests in Appendix B made with several phase screens constructed by adjusting the multiplying factor.

We used a complex Hermitian symmetric spectrum to make the phase field $\phi_{K}(m, n)$ real, i.e. The complex coefficients of Eq. (4) are conjugate by applying $\psi(k, l)=-\psi(-k,-l)$ in the construction of the screen. Equation (4) is suitable for computation by FFT where the elements of the input complex array are the coefficients $c(k, l)=-c(-k,-l)=\left(k^{2}+l^{2}\right)^{-\beta / 4} \mathrm{e}^{ \pm \mathrm{i} \psi(k, l)}$. We generate a random phase field $\phi_{K}(m, n)$ by making the Fourier phase $\psi(k, l)$ a random variable uniformly distributed over $[0,2 \pi]$ (Rice 1944, p. 287). We had to make the adjustment factor $b=2$ in Eq. (4) so that the phase structure function $D_{\phi}(r)$ computed directly from the screen yields a coherence length that matches the theoretical value $s_{0}$. Coles et al. (1995) have devised a method to randomize a phase screen. Instead of our complex numbers $b \mathrm{e}^{-\mathrm{i} \psi(k, l)}$ in Eq. (4), with the random phase $\psi(k, l)$ described above, Coles et al. (1995) set complex random numbers made of independent zero-mean Gaussian random variables $x$ and $y$ with variance $\sigma^{2}$ for their real and imaginary parts. In polar coordinates, these variables $x$ and $y$ have magnitudes following a Rayleigh probability distribution and phases distributed uniformly over $[0,2 \pi]$ as for our $\psi(k, l)$ (see Thompson et al. 1986, pp. 259-260; and reference to Papoulis 1956). We have verified in generating independent zero-mean Gaussian random variables $x$ and $y$ of various $\sigma$ that the mean amplitude of the corresponding Rayleigh probability distribution for $\sigma=1.6$ is 2 , i.e. the value of our $b$ factor. Thus, the method we use to generate the random complex numbers in Eq. (4) is a satisfactory approximation of the formal synthesis of the random phase field $\phi_{K}$ in Coles et al. (1995).

The intensity of the pulsar $i\left(\boldsymbol{x}^{\prime}, \lambda\right)=|\boldsymbol{E}|^{2}\left(\boldsymbol{x}^{\prime}, \lambda\right)$ at wavelength $\lambda$ is computed with the discrete form of the Kirchhoff-Fresnel integral where the gridded phase screen $\phi_{K}(m, n)$ substitutes the continuous Kolmogorov phase $\phi_{K}(\boldsymbol{x})$. The electric field (Eq. (1)) at the grid position $p$ of the radiotelescope and wavelength $\lambda$ is:

$E(p, \lambda)=\frac{E_{\mathrm{s}} \mathrm{d} x_{\mathrm{s}}^{2}}{\lambda L} \sum_{n=1}^{n=H} \sum_{m=1}^{m=H} \mathrm{e}^{\mathrm{i}\left[\frac{2 \pi}{\lambda} \ell(p, m, n)+\phi_{K}(m, n)\right]}$

where the geometric path $\ell(p, m, n)$ is from the point source pulsar $P$ through the phase screen at the running pixel $S(m, n)$ to the radiotelescope position $T$ along the $x$-axis $(p)$ in Fig. 1. We have not approximated this path by a power series in our code so that we assumed neither the Fraunhofer approximation nor the Fresnel approximation but carried out the full computation of $\ell(p, m, n)$. The parameter $\mathrm{d} x_{\mathrm{s}}$ is the spatial resolution of the phase screen which does not have to be equal to the grid step $\Delta r$ controlling the limits $q_{\min }$ and $q_{\max }$ of the Kolmogorov spectrum $P_{3 N}$. Since Eq. (5) is the discrete form of Eq. (1), the parameter $H$ of Eq. (5) controls the size of the integration surface noted $\mathcal{S}$ in Eq. (1); $\mathcal{S}=\left(H \times \mathrm{d} x_{\mathrm{s}}\right)^{2}$. 


\section{Construction of a large square kolmogorov phase screen}

We have computed a relatively large Kolmogorov phase screen for the pulsar PSR1937+21 with Eq. (4) on an alpha server assuming turbulence strength $C_{n}^{2}=10^{-3} \mathrm{~m}^{-20 / 3}$ and screen thickness $z$ to be the pulsar distance $(3.6 \mathrm{kpc})$ (this assumption is discussed below). The size of the screen is $N=2^{17}$, i.e. there are $\sim 131 \mathrm{k} \times 131 \mathrm{k}$ pixels. Obviously, this computation could not be done in a single 2D FFT and we had to resort to multiple 1D FFT's. Each line of the input complex array, coefficients $c(k, l)$ of Eq. (4), was first transformed by a $2^{17}$ point 1D FFT along the $x$-axis. Then, each resulting column was transformed by a $2^{17}$ point $1 \mathrm{D}$ FFT along the $y$-axis. The construction of this large square phase screen took 10 days on our workstation and needs 68 GigaBytes of disk space to store all the phases but the peak storage during the computation was 200 GigaBytes.

With the assumed turbulence strength $C_{n}^{2}=10^{-3} \mathrm{~m}^{-20 / 3}$ and thickness $z=3.6 \mathrm{kpc}$, the coherence length in the screen is $s_{0}=$ $2.6610^{7} \mathrm{~m}$ at $1.41 \mathrm{GHz}$ as calculated from the phase structure function $D_{\phi}\left(s_{0}\right)=1 \mathrm{Rd}^{2}$ with the theoretical expression $D_{\phi}(s)=$ $8 \pi r_{\mathrm{e}}^{2} \lambda^{2} C_{N}^{2} z f(\alpha)(\alpha+1)^{-1} s^{\alpha}$, where numerically $f(\alpha)=1.12$ for $\alpha=5 / 3$ (Armstrong et al. 1995). To qualify our phase screen, we have computed the phase structure functions $D_{\phi}(s)$ along multiple $x$-lines through the screen and, orthogonally, along $y$. From the log-log plots of these functions (examples in Fig. 2), we made linear least-square-fits and found slopes of $1.68 \pm 0.10$ for $x$ and $1.64 \pm 0.1$ for $y$. The theoretical slope of $D_{\phi}(s)$ is $\alpha=5 / 3$ for an isotropic turbulent medium with the exponent $\beta=11 / 3$ in $P_{3 N}$. We have also measured the coherence lengths from these phase structure functions: $r_{\text {coh }}=2.4 \pm 0.6 \times 10^{7} \mathrm{~m}$ along $x$ and $r_{\text {coh }}=3.2 \pm 0.6 \times 10^{7} \mathrm{~m}$ along $y$. These values are consistent with the value $s_{0}=2.66 \times 10^{7} \mathrm{~m}$ predicted by the structure function $D_{\phi}\left(s_{0}\right)=1 \mathrm{Rd}$. This is also consistent with the value obtained from the equation $r_{\text {coh }}=V / t_{\mathrm{d}} \simeq 2.1 \times 10^{7} \mathrm{~m}$, where $V=50 \mathrm{~km} \mathrm{~s}^{-1}$ and $t_{\mathrm{d}}=7 \mathrm{~min}$ is the observed diffractive timescale toward the pulsar B1937+21 (Ryba 1991). We note that it is coincidental that $s_{0}$ has the expected value by using the conventional value of $C_{n}^{2}$ for PSR1937+21 and our assumption above that the thickness $z$ equals the pulsar distance.

\section{Results: simulation of the scintillation of the pulsar B1937+21 at 1.41 and $1.7 \mathrm{GHz}$}

Prior to the full scale simulations, we studied the convergence of the computation of the dynamic spectra that depends on four parameters: the size $S$ of the integration surface of Eq. (1), the spatial resolution $\mathrm{d} x_{\mathrm{s}}$ to read the phase screen file, the grid step $\Delta r$ in the phase screen and the size $N$ of the phase screen (Eq. (4)) which control the minimum and maximum spatial frequencies $q_{\min }=\frac{1}{N \Delta r}$ and $q_{\max }=\frac{1}{2 \Delta r}$ of the Kolmogorov spectrum $P_{3 N}$. The parameter $N$ was fixed to $2^{17}$, limited only by the maximum disc space available with our computer to store the phase screen. This screen size corresponds to a period of 6 months, for an observer line of sight sliding across the screen with a velocity of $50 \mathrm{~km} \mathrm{~s}^{-1}$. We have empirically determined the other three parameters by a sequence of tests that are described in detail in Appendix B.

In Fig. 3, we present the intensity of the pulsar B1937+21 simulated during this period of six months at 1.41 and $1.7 \mathrm{GHz}$. The intervening phase screen $\phi_{K}(m, n)$ is constructed with $C_{n}^{2}=$ $10^{-3} \mathrm{~m}^{-20 / 3}$ and $z=3.6 \mathrm{kpc}$ that are the parameters of B $1937+21$ (Ryba 1991; Johnston et al. 1998), $N=2^{17}$ and $\Delta r=s_{0} / 4$ in

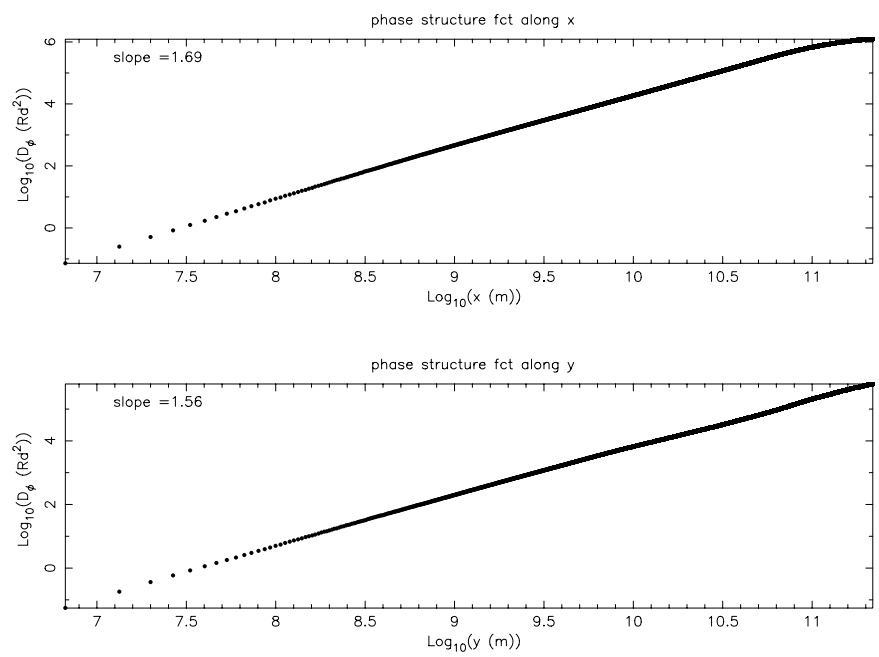

Fig. 2. Examples of the phase structure functions $D_{\phi}(x)$ and $D_{\phi}(y)$ computed for 1D cuts along the $x$ and $y$ axis across the whole $131 \mathrm{k} \times 131 \mathrm{k}$ phase screen $\phi_{K}(m, n)$ constructed with $C_{n}^{2}=10^{-3}, z=3.6 \mathrm{kpc}$ and $\Delta r=s_{0}$. The slopes of these structure functions measured from the log$\log$ plots are satisfactorily close to the theoretical value $\alpha=5 / 3$ for $\beta=11 / 3$ of the Kolmogorov spectrum $P_{3 N}$.

Eq. (4) based on our conclusion in the second test (Appendix B). The coherence length in the phase screen is $2.6 \times 10^{7} \mathrm{~m}$ thus the lower and upper limit scales of the Kolmogorov spectrum $P_{3 N}$ are $q_{\min }=\frac{1}{2^{17} \times 2.6 \times 10^{7}}=\frac{1}{3.4 \times 10^{12}} \mathrm{~m}^{-1}$ and $q_{\max }=\frac{1}{2 \times 2.6 \times 10^{7}} \mathrm{~m}^{-1}$. We adopt the integration surface $S=\left(4 r_{\mathrm{S}}\right)^{2}$ to compute Eq. (5) from the conclusion in the first test (Table 1). We use the resolution $\mathrm{d} x_{\mathrm{s}}=s_{0} / 4$ (Table 2) to read the phase screen file into Eq. (5). This resolution is conservative relative to our conclusion in the third test; it was dictated for algorithmic peculiarities to the expense of computing speed. A dynamic spectrum was computed in these conditions every other 1.25 days. Averaging these dynamic spectra provides the intensity measured by the telescope over the integration time $70 \mathrm{~min}$ and the bandwidth $8.8 \mathrm{MHz}$, sampled over $32 \times 32$ time bins and frequency channels in our computation. The velocity of the screen is $V=50 \mathrm{~km} \mathrm{~s}^{-1}$. This simulation took twice 1.5 months on our alpha server.

Overall, Fig. 3 shows the typical behavior of flux density recorded for pulsars; for instance B1937+21 at Nançay at 1.41 and $1.7 \mathrm{GHz}$ in Figs. 1 and 2 of LRC98. In addition, there is the interesting feature labeled "ESE" and present simultaneously at 1.41 and $1.7 \mathrm{GHz}$ in Fig. 3 from day 30 to day 40. The significant drop of flux density ( $60 \%$ below the mean value) and the low rms during this ESE period ( $\mathrm{rms}=0.10$ during the ESE versus $\mathrm{rms}=0.71$ off ESE) for these 10 days is alike the ESE observed toward B1937+21 in October 1989 (Cognard et al. 1993). In this previous paper, we suggested this phenomenon was caused by a discrete cloud of plasma following the standard interpretation of Fiedler et al. (1987). Our simulation shows instead that this event can arise naturally because of the turbulence in the ionized interstellar medium as conjectured. We have labeled this event "ESE" in Fig. 3. The ratio of the duration of this event to the length of our simulation is $\sim 10$ days/180 days $\simeq 5 \%$. Although this might be fortuitous, this percentage is consistent with the observations of B1937+21 at Nançay for which the rate of occurrence of "ESE's" is 4\% (LRC98). Figure 4 shows the cross correlation function of the flux densities at 1.41 and $1.7 \mathrm{GHz}$ (Fig. 3). The zero-lag value $\simeq 0.8$ is consistent with the observed value $0.93 \pm 0.05$ (LRC98). 
Intensity at $1.4 \mathrm{GHz}$

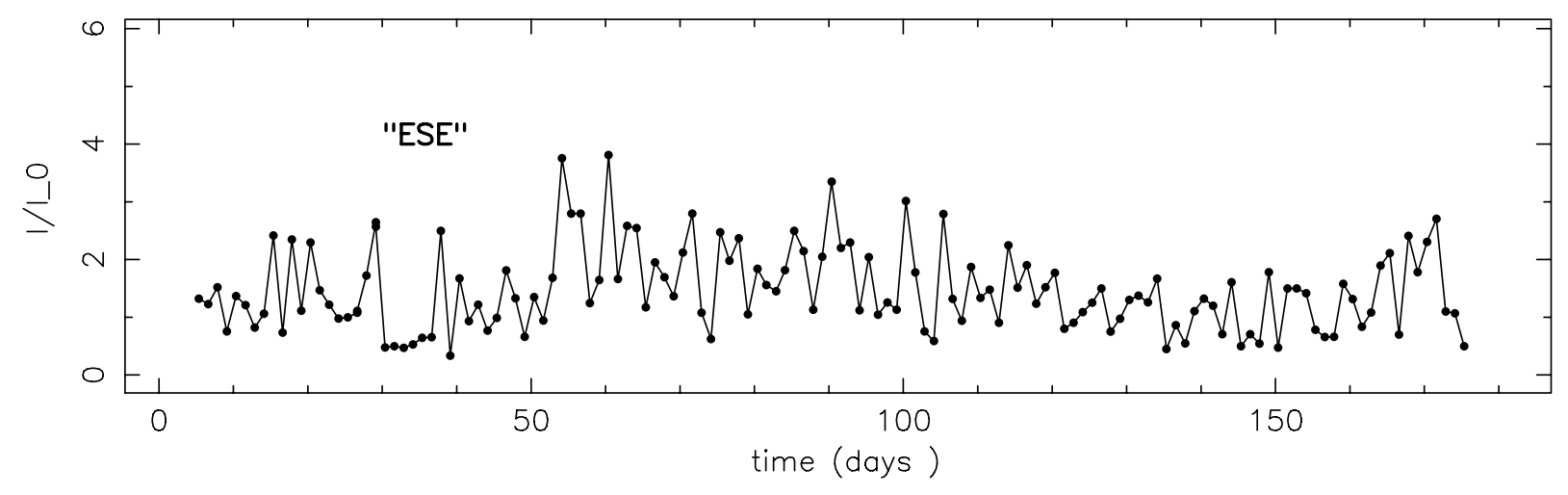

Intensity at $1.7 \mathrm{GHz}$

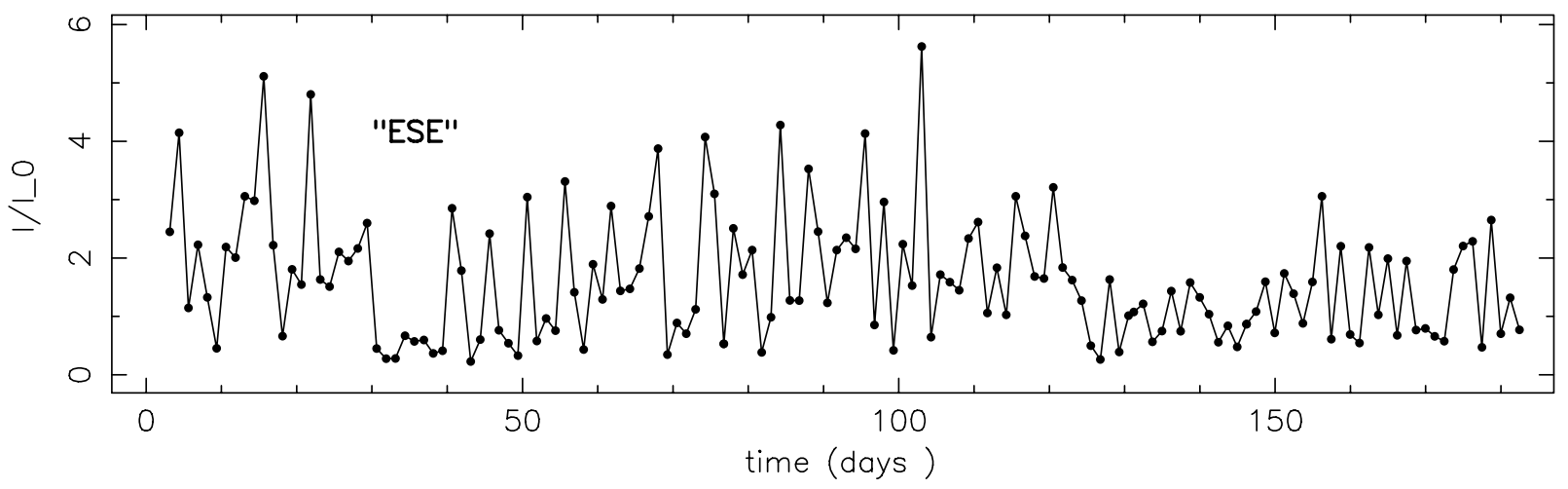

Fig. 3. Intensity of B1937+21 simulated at $1.41 \mathrm{GHz}$ and $1.7 \mathrm{GHz}$. The intervening phase screen is constructed with $C_{n}^{2}=10^{-3}, z=3.6 \mathrm{kpc}$, $N=2^{17}$ and $\Delta r=s_{0} / 4$ in Eq. (4) and $S=\left(4 r_{\mathrm{S}}\right)^{2}$ and $\mathrm{d} x_{\mathrm{s}}=s_{0} / 4$ in Eq. (5). Between days 30 and 40, there is an event occurring simultaneously at 1.41 and $1.7 \mathrm{GHz}$ which is alike the behavior of the flux density recorded in direction of B1937+21 at Nançay in October 1989 and interpreted as an Extreme Scattering Event. The mean intensities $\langle I\rangle$ are 1.46 and 1.60 and the modulation indexes $m$ are 0.49 and 0.67 for these two series.

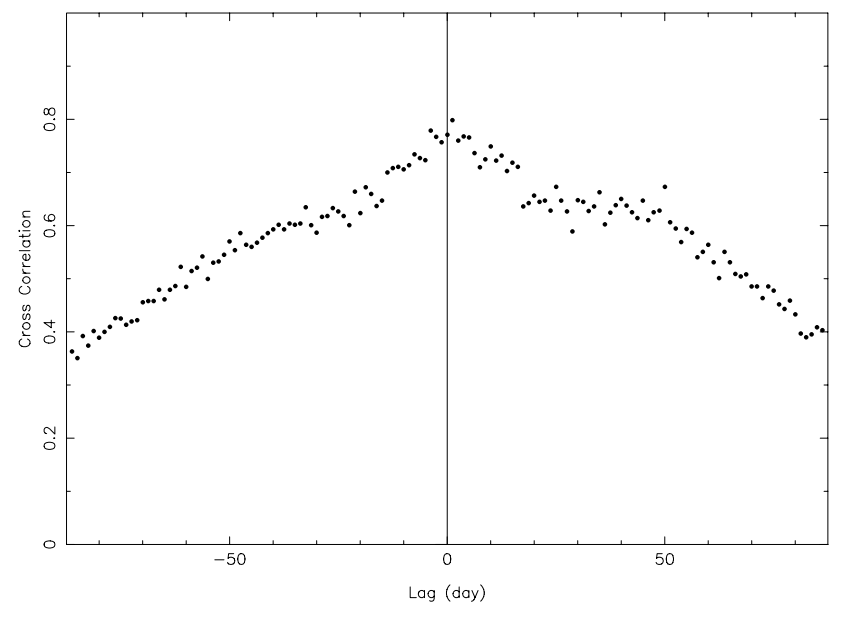

Fig. 4. Cross-correlation function of the flux densities at $1.41 \mathrm{GHz}$ and $1.7 \mathrm{GHz}$ of Fig. 3.

The duration of the event labeled "ESE" in our simulation is comparable to the event observed at Nançay in October 1989 (12 days) but is short compared to three others of duration 13 months identified in the radio light curves of B1937+21 simultaneously at 1.41 and $1.7 \mathrm{GHz}$ by LRC98. The extraordinary focusing of pulsar waves is caused by the large scale fluctuations of the electronic density in the interstellar medium. The largest fluctuation scale in our simulation is $2^{17} \times 2.6 \times 10^{7} / 4 \sim 10^{12} \mathrm{~m}$ while the ionized interstellar medium is made of fluctuations up to $10^{18} \mathrm{~m}$ (Armstrong et al. 1995). We expect that longer "ESE"s require to include fluctuations of larger scales in our simulation. We plan to extend this calculation on a supercomputer. We note here that the relatively long events labeled "ESE" in Hamidouche et al. (2002) are from a simulation that was improperly done with an integration surface as small as $S=\left(0.7 r_{\mathrm{S}}\right)^{2}$ chosen before we made the tests of the present paper.

Finally, we note that the anti-correlation coefficient -0.62 between flux density and TOA in the simulation conducted with a rectangular phase screen is reported in Hamidouche (2003), and is comparable to the correlation coefficient -0.43 to -0.73 depending on data segments of the Nançay observations in LRC98. We have not calculated this correlation in the final simulation.

\section{Conclusion}

We have presented the simulation of the scintillation of pulsars carried out within the frame of Physical Optics by extending the seminal work by Cordes et al. (1986). We have built a large square Kolmogorov phase screen of $131 \mathrm{k} \times 131 \mathrm{k}$ pixels on an alpha server and shown how to set properly the parameters of the Kirchhoff-Fresnel integral by conducting several tests. Our final simulation was done for the condition of turbulence known in the direction of the pulsar B1937+21 $\left(C_{n}^{2} \sim 10^{-3}\right.$ and distance 
$z=3.6 \mathrm{kpc}$ ) and the radio light curves were generated at 1.41 and $1.7 \mathrm{GHz}$ for a period of 6 months.

These two light curves exhibit simultaneous variations at 1.41 and $1.7 \mathrm{GHz}$ that are alike the "ESE" observed at these frequencies at Nançay in October 1989 and that lasted $\sim 10$ days. Our simulation shows that this observed event can be caused naturally by the turbulence in the ionized interstellar medium instead of invoking the crossing of a discrete over pressured ionized cloud on the line of sight as in the model of Fiedler et al. (1987). We think that longer events could occur by including the electronic density fluctuations of larger scales in the construction of the Kolmogorov phase screen used in the simulation.

Finally, we note that Deshpande (2000) stresses that the opacity differences in $\mathrm{HI}$ and other species measured over a transverse separation $x_{0}$ result from all scale of the 3D power spectrum of the opacity fluctuations while they are currently, and erroneously in his opinion, interpreted as over pressured and overdensed cloudlets of size $x_{0}$. Our simulation of "ESE's" caused by the turbulent ionized interstellar medium strengthens this opinion for the neutral gas.

Acknowledgements. We are grateful to our referee Prof. Barney Rickett who carefully read the manuscript and provided many valuable suggestions to improve the paper. We are in debt to the legacy of Max Born and Emil Wolf. We would like to thank Françoise Combes and François Lefeuvre for useful discussions. M.H. would like to thank, Richard Crutcher, the director of the Laboratory for Astronomical Imaging at the University of Illinois.

\section{Appendix A: Construction of a Kolmogorov phase screen}

The definition of the 2D phase power spectrum of the phase field $\phi(x, y)$ averaged over the rectangular screen surface $N M \Delta r^{2}$ is:

$P_{2 \phi}\left(q_{x}, q_{y}\right)=\frac{\left|\mathcal{F}_{2 \phi}\left(q_{x}, q_{y}\right)\right|^{2}}{N M \Delta r^{2}}$

where $\mathcal{F}_{2 \phi}\left(q_{x}, q_{y}\right)$ is the Fourier Transform of $\phi(x, y)$. Hence, the module of the Fourier component for the frequencies $\left(q_{x}, q_{y}\right)$ is:

$\left|\mathcal{F}_{2 \phi}\left(q_{x}, q_{y}\right)\right|=\sqrt{N M \Delta r^{2} P_{2 \phi}\left(q_{x}, q_{y}\right)}$

Lovelace (1970) and Lovelace et al. (1970) have established:

$P_{2 \phi}\left(q_{x}, q_{y}\right)=2 \pi z\left(\lambda r_{\mathrm{e}}\right)^{2} P_{3 \mathrm{~N}}\left(q_{x}, q_{y}, q_{z}=0\right)$.

Hence, the complex Fourier components of the phase field $\phi(x, y)$ are:

$\mathcal{F}_{2 \phi}\left(q_{x}, q_{y}\right)=\sqrt{2 \pi} \lambda r_{\mathrm{e}} \sqrt{N M \Delta r^{2} z C_{n}^{2}\left(q_{x}^{2}+q_{y}^{2}\right)^{-\beta / 2}} \mathrm{e}^{-\mathrm{i} \psi\left(q_{x}, q_{y}\right)}(\mathrm{A} .4)$

that must satisfy the complex Hermitian symmetric spectrum with the Fourier phase $\psi\left(q_{x}, q_{y}\right)=-\psi\left(-q_{x},-q_{y}\right)$ to make the phase field $\phi(x, y)$ real. $\psi\left(q_{x}, q_{y}\right)$ is a random variable uniformly distributed over [0,2 $]$ as prescribed by Rice (1944, p. 287). $C_{n}^{2}$ is the turbulence strength and $z$ is the propagation length.

The phase field $\phi(x, y)$ can be computed by the inverse Fourier Transform of $\mathcal{F}_{2 \phi}\left(q_{x}, q_{y}\right)$. Although $P_{3 N}$ is a power-law, the Fourier integral is finite because there is an outer scale in the ionized interstellar medium that makes $P_{3 N}$ becomes zero for small $q$ rather than infinity when $q \rightarrow 0$. Also, we point out that there is the factor $(2 \pi)^{-2}$ in this integral to be consistent with the Fourier Transform definition used to define $P_{3 N}$ in Rickett (1977, Eq. (6)) and used to demonstrate the Lovelace
Table B.1. Sensitivity of the averaged intensity and modulation index to the integration surface at $1.4 \mathrm{GHz}^{a}$.

\begin{tabular}{llll}
\hline \hline $\begin{array}{l}\text { Integration } \\
\text { surface } S\end{array}$ & $\begin{array}{l}\text { Nber of } \\
\text { intensities }\end{array}$ & $\langle I\rangle$ & Index m \\
\hline$\left(1 r_{\mathrm{S}}\right)^{2}$ & 303 & 0.28 & 0.95 \\
$\left(2 r_{\mathrm{S}}\right)^{2}$ & 302 & 0.83 & 0.80 \\
$\left(4 r_{\mathrm{S}}\right)^{2}$ & 299 & 1.39 & 0.63 \\
$\left(6 r_{\mathrm{S}}\right)^{2}$ & 296 & 1.52 & 0.59 \\
$\left(8 r_{\mathrm{S}}\right)^{2}$ & 292 & 1.55 & 0.58 \\
$\left(10 r_{\mathrm{S}}\right)^{2}$ & 289 & 1.56 & 0.58 \\
\hline
\end{tabular}

${ }^{a} \Delta r=\mathrm{d} x_{\mathrm{s}}=s_{0}$ kept fixed.

relationship (Lovelace 1970, Eq. (36)). This inverse Fourier Transform is:

$$
\begin{aligned}
& \phi(x, y)=(2 \pi)^{-2+\frac{1}{2}} \lambda r_{\mathrm{e}} \Delta r \sqrt{N M z C_{N}^{2}} \\
& \times\left[\int_{-q_{x, \max }}^{-q_{x, \min }} \int_{-q_{y, \max }}^{-q_{y, \text { min }}}\left(q_{x}^{2}+q_{y}^{2}\right)^{-\beta / 4} \mathrm{e}^{-\mathrm{i} \psi\left(q_{x}, q_{y}\right)} \mathrm{e}^{-\mathrm{i}\left(q_{x} x+q_{y} y\right)} \mathrm{d} q_{x} \mathrm{~d} q_{y}\right. \\
& \left.\quad+\int_{+q_{x, \min }}^{+q_{x, \max }} \int_{+q_{y, \text { min }}}^{+q_{y, \max }}\left(q_{x}^{2}+q_{y}^{2}\right)^{-\beta / 4} \mathrm{e}^{-\mathrm{i} \psi\left(q_{x}, q_{y}\right)} \mathrm{e}^{-\mathrm{i}\left(q_{x} x+q_{y} y\right)} \mathrm{d} q_{x} \mathrm{~d} q_{y}\right] .
\end{aligned}
$$

This integral was split into two parts to avoid the singularity of the power-law function. In Eq. (A.5) for the rectangular screen case, the minimum frequencies are $q_{x, \min }=\frac{1}{N \Delta r}$ and $q_{y, \min }=\frac{1}{M \Delta r}$.

\section{Appendix B: Tests for the computation of the dynamic spectra}

Prior to the full scale simulations, we studied the convergence of the computation of the dynamic spectra that depends on four parameters: the size $S$ of the integration surface $\mathcal{S}$ of Eq. (1), the spatial resolution $\mathrm{d} x_{\mathrm{s}}$ to read the phase screen file, the grid step $\Delta r$ in the screen phase, and the size $N$ of the phase screen (Eq. (4)). These latter two parameters control the minimum and maximum spatial frequencies $q_{\min }=\frac{1}{N \Delta r}$ and $q_{\max }=\frac{1}{2 \Delta r}$ of the Kolmogorov spectrum $P_{3 N}$. The parameter $N$ is fixed to $2^{17}$ in our computation and we have empirically determined the other three parameters.

As a first test, we study the effect of $S$ of Eq. (1) while keeping the parameters $\Delta r$ and $\mathrm{d} x_{\mathrm{s}}$ fixed and equal to $s_{0}$. We express the dimension of the integration surface $S$ in terms of the scattering radius $r_{\mathrm{S}}$ since, as shown below, the required $S$ to make the dynamic spectra numerically convergent is a few times this scale. In fact, Coles et al. (1995) have demonstrated that the integration surface in the phase screen has to be larger than the scattering disk. The physical idea is that the scattering disk is the approximate area in the phase screen irradiating a single location in the observing plane. We chose the following sizes $S$ for our test: $S=\left(1 r_{\mathrm{S}}\right)^{2}, S=\left(2 r_{\mathrm{S}}\right)^{2}, S=\left(4 r_{\mathrm{S}}\right)^{2}, S=\left(6 r_{\mathrm{S}}\right)^{2}, S=\left(8 r_{\mathrm{S}}\right)^{2}$, $S=\left(10 r_{\mathrm{S}}\right)^{2}$. For each size $S$, we compute the whole series of dynamic spectra at $1.41 \mathrm{GHz}$ by shifting $\mathcal{S}$ by 2.5 days across the screen using $V=50 \mathrm{~km} \mathrm{~s}^{-1}$. Each dynamic spectrum is $70 \mathrm{~min} \times 8.8 \mathrm{MHz}$ in size, similarly to observations of B1937+21 at Nançay, and sampled over $8 \times 8$ pixels. For each series, we derive the mean intensity $\langle I\rangle$ and the modulation index $m=\frac{I_{\mathrm{rms}}}{\langle I\rangle}$ in Table B.1. This table shows that these two indicators converge 


\section{Intensity}

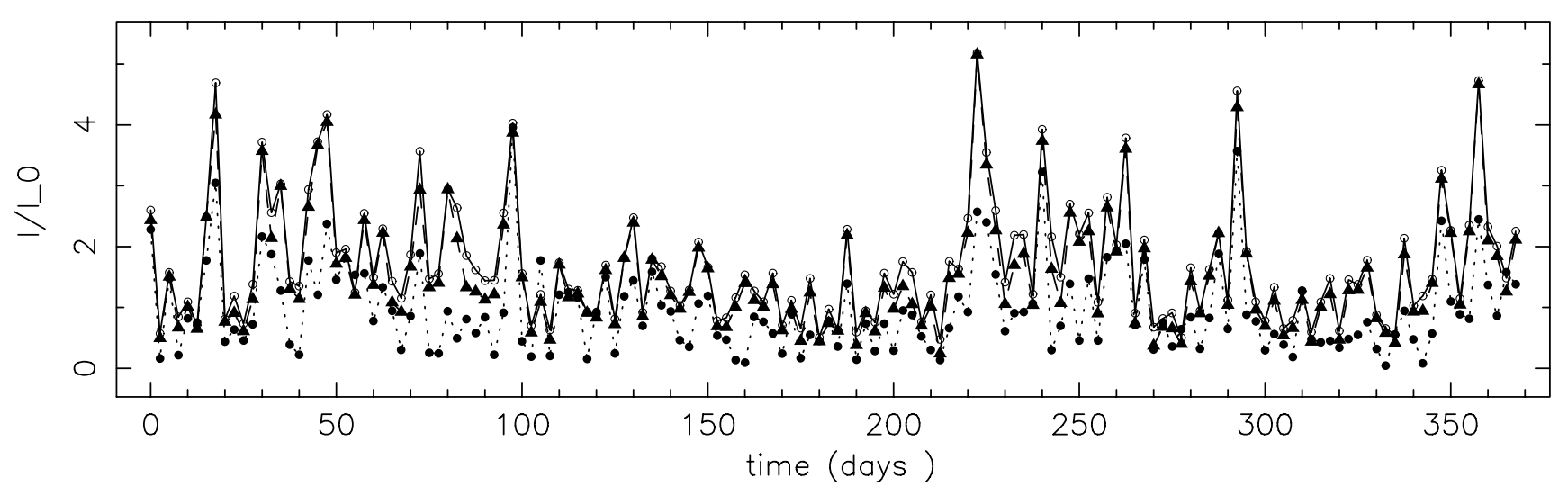

Intensity

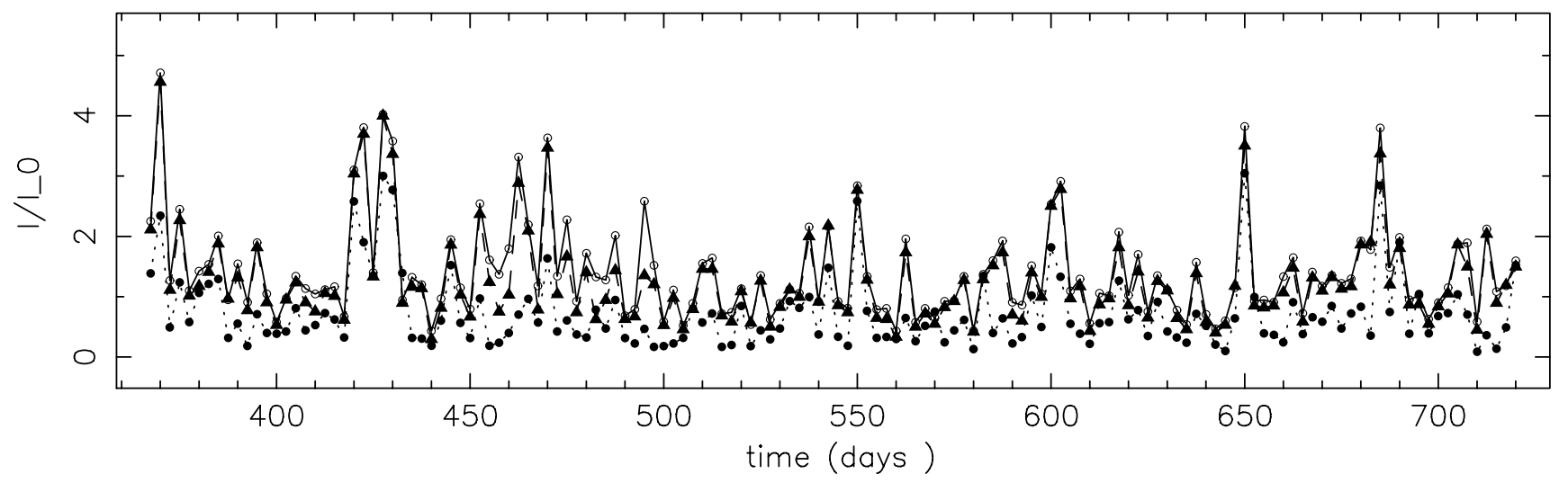

Fig. B.1. Test on the parameter $S$ : simulations of the intensity of a pulsar at $1.41 \mathrm{GHz}$ every other 2.5 days observed through the phase screen $\phi_{K}(m, n)$ constructed with $C_{n}^{2}=10^{-3}, z=3.6 \mathrm{kpc}, N=2^{17}$ and $\Delta r=s_{0}$. The spatial resolution used to read the screen file is $\mathrm{d} x_{\mathrm{s}}=\Delta r$. The three light curves are for the integration surfaces $S=\left(2 r_{\mathrm{S}}\right)^{2}$ (filled circle), $S=\left(4 r_{\mathrm{S}}\right)^{2}$ (filled triangle), $S=\left(10 r_{\mathrm{S}}\right)^{2}$ (empty circle). This test demonstrates that the computation of the dynamic spectra has converged for the integration surface $S=\left(4 r_{\mathrm{S}}\right)^{2}$.

when $S$ reaches $\left(4 r_{\mathrm{S}}\right)^{2}$. In Fig. B.1, we show only three series for clarity; $S=\left(2 r_{\mathrm{S}}\right)^{2}, S=\left(4 r_{\mathrm{S}}\right)^{2}$ and $S=\left(10 r_{\mathrm{S}}\right)^{2}$ to depict the convergence process. In addition, we illustrate this convergence process in Fig. B. 2 by showing dynamic spectra computed at the same position in the observer plane but for the 6 integration surfaces mentioned above. From this first test summarized by Table B.1, Figs. B.1 and B.2, we conclude that convergence of dynamic spectra starts for $S=\left(4 r_{\mathrm{S}}\right)^{2}$ since improvement in the average intensity and index $m$ are less than $10 \%$ for larger surfaces $S$.

As a second test, we study the impact of the grid step $\Delta r$ in the screen phase while keeping fixed $S=\left(4 r_{\mathrm{S}}\right)^{2}$ and $\mathrm{d} x_{\mathrm{s}}=$ $s_{0}$. The parameter $\Delta r$ is directly related to the minimum and maximum spatial frequencies $q_{\mathrm{min}}=\frac{1}{N \Delta r}$ and $q_{\max }=\frac{1}{2 \Delta r}$ of the Kolmogorov spectrum $P_{3 N}$ as already mentioned. Note that $\Delta r$ appears conveniently in the multiplying factor of the phase screen $\phi_{K}(m, n)$ in Eq. (4) and hence it can be easily changed to any value to modify $q_{\max }$ and, correlatively, $q_{\min }$. The parameter $N$ is not amendable after the double summation of Eq. (4) has been calculated and stored in a computer file. In principle, we would have liked to tune $N$ in order to keep $q_{\min }=$ $\frac{1}{N \Delta r}$ unchanged while $\Delta r$ is adjusted to increase $q_{\max }=\frac{1}{2 \Delta r}$. One expects that the high frequency part of the Kolmogorov spectrum becomes insignificant in shaping the dynamic spectrum when $q_{\max }$ is sufficiently high while the low frequency part modifies it drastically. This test is difficult to implement in practice because it requires computing several screens with different $N$ while keeping the same random phases $\psi(k, l)$ for the lower part of the spectrum. Instead of this approach, we simulate the effect by superimposing a corrugated surface of period $q^{-1}$ and amplitude $\delta \phi$ upon the original screen. The aim is to seek which perturbating surface $(q, \delta \phi)$ degrades significantly the dynamic spectrum when compared to the one computed with the original phase screen. This test covers the following corrugated surfaces: $\delta \phi=101^{\circ}$ and $q=\frac{1}{2 \times 2 s_{0}} ; \delta \phi=57^{\circ}$ and $q=\frac{1}{2 s_{0}} ; \delta \phi=32^{\circ}$ and $q=\frac{1}{2 s_{0} / 2} ; \delta \phi=18^{\circ}$ and $q=\frac{1}{2 s_{0} / 4} ; \delta \phi=10^{\circ}$ for $q=\frac{1}{2 s_{0} / 8}$. These amplitudes $\delta \phi$ are derived from the phase structure function $D_{\phi}(s)$ for the separations $s=2 s_{0}, s=s_{0}, s=s_{0} / 2, s=s_{0} / 4$ and $s=s_{0} / 8$. Figure B.3 shows the comparison of the dynamic spectra computed with these five perturbations $(q, \delta \phi)$ as well as the dynamic spectrum simulated with the original screen $(\delta \phi=0)$ constructed with $C_{n}^{2}=10^{-3}, z=3.6 \mathrm{kpc}, N=2^{17}$ and $\Delta r=s_{0} / 4$. The morphology and averaged intensities of these dynamic spectra indicate that perturbations are significant above $\left(\delta \phi=32^{\circ}, q=\frac{1}{2 s_{0} / 2}\right)$, i.e. $q_{\max }$ must be at least as high as $\frac{1}{2 s_{0} / 2}$, i.e. $\Delta r \leq s_{0} / 2$, for convergence of the computation. 

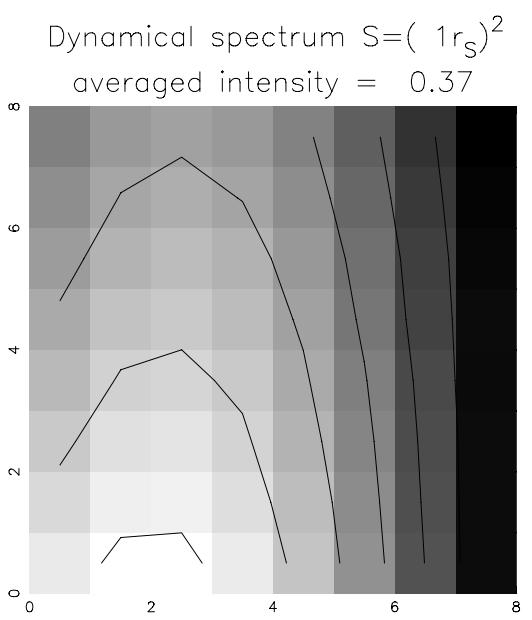

Dynamical spectrum $S=\left(6 r_{S}\right)^{2}$

averaged intensity $=2.57$

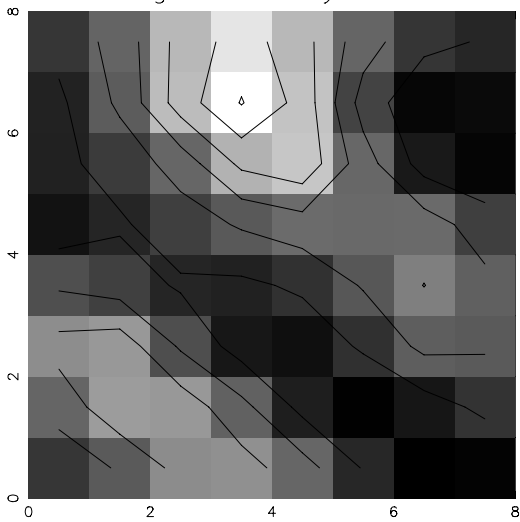

Dynamical spectrum $S=\left(2 r_{S}\right)^{2}$

averaged intensity $=2.28$

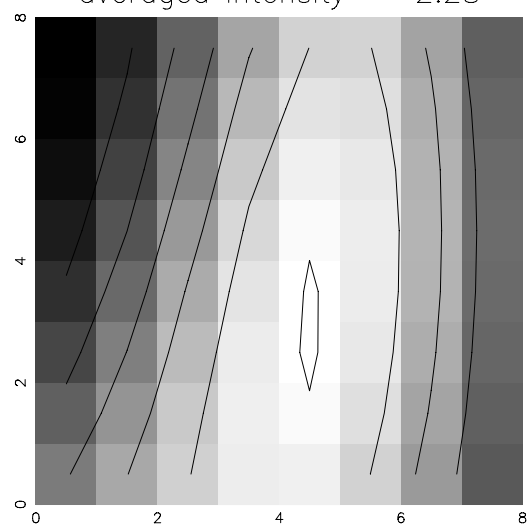

Dynamical spectrum $S=\left(8 r_{S}\right)^{2}$

averaged intensity $=2.59$

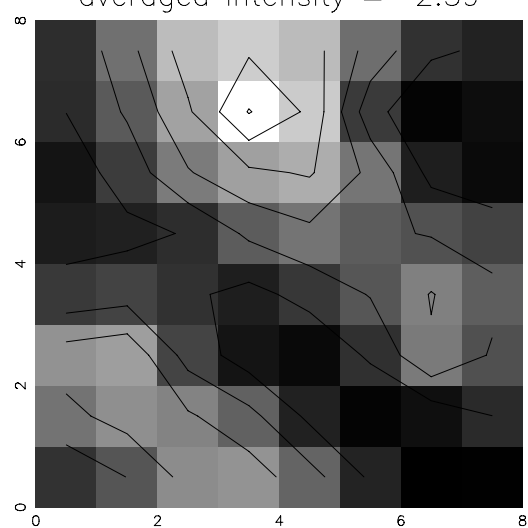

Dynamical spectrum $S=\left(4 r_{S}\right)^{2}$

averaged intensity $=2.44$

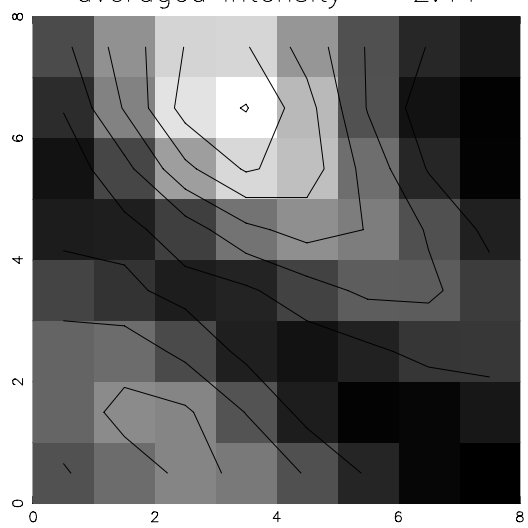

Dynamical spectrum $S=\left(10 r_{s}\right)^{2}$

averaged intensity $=2.61$

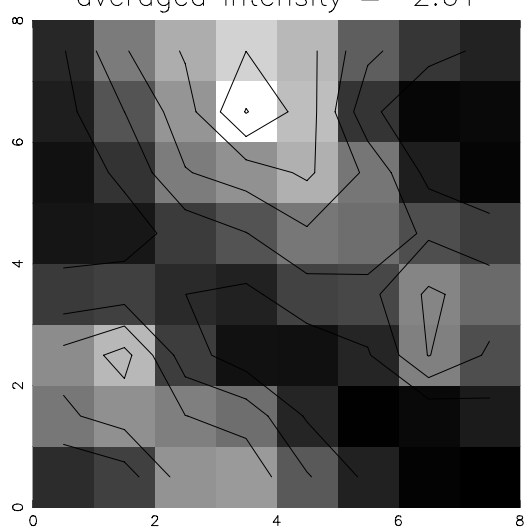

Fig. B.2. Test on parameter $S$ : dynamic spectra computed, at $1.41 \mathrm{GHz}$, at the same telescope location but for different sizes $S$ of the integration surface $\mathcal{S}$ in Eq. (1). The six cases shown are for $S=\left(1 r_{\mathrm{S}}\right)^{2},\left(2 r_{\mathrm{S}}\right)^{2},\left(4 r_{\mathrm{S}}\right)^{2},\left(6 r_{\mathrm{S}}\right)^{2},\left(8 r_{\mathrm{S}}\right)^{2},\left(10 r_{\mathrm{S}}\right)^{2}$. The total size of the dynamic spectrum is $70 \mathrm{~min} \times 8.8 \mathrm{MHz}$ sampled over $8 \times 8$ pixels, i.e. unity corresponds to $1.1 \mathrm{MHz}$ along the frequency axis (vertical) and to 8.8 min along the time axis (horizontal) with the screen speed $V=50 \mathrm{~km} \mathrm{~s}^{-1}$. The phase screen $\phi_{K}(m, n)$ is constructed as in Fig. 2 . This figure shows that the dynamic spectrum converges from the surface size $S=\left(4 r_{\mathrm{S}}\right)^{2}$.

Table B.2. Sensitivity of the averaged intensity and modulation index to $q_{\max }{ }^{a}$.

\begin{tabular}{lccccc}
\hline \hline$\Delta r$ & $q_{\max }=\frac{1}{2 \Delta r}$ & $\begin{array}{c}\text { Sampling } \\
\text { of dyn. sp. }\end{array}$ & $\begin{array}{c}\text { Nb of } \\
\text { intensities }\end{array}$ & $\langle I\rangle$ & Index $m$ \\
\hline$s_{0}$ & $\frac{1}{2 s_{0}}$ & $8 \times 8$ & 299 & 1.39 & 0.63 \\
$s_{0} / 2$ & $\frac{1}{2 s_{0} / 2}$ & $16 \times 16$ & 142 & 1.43 & 0.54 \\
$s_{0} / 4$ & $\frac{1}{2 s_{0} / 4}$ & $32 \times 32$ & 69 & 1.58 & 0.50 \\
\hline
\end{tabular}

${ }^{a} \Delta r=\mathrm{d} x_{\mathrm{s}}=s_{0}$ kept fixed.

We complement this test done at a single position of the observer by simulating three intensity series across the full screen with the grid step $\Delta r=\mathrm{d} x_{\mathrm{s}}$ set to $1 s_{0}, s_{0} / 2$ and $s_{0} / 4$. The dynamic spectra are sampled over $8 \times 8,16 \times 16,32 \times 32$ pixels, respectively, to synthesize the same $70 \mathrm{~min} \times 8.8 \mathrm{MHz}$ domain. Table B. 2 shows that the mean intensity $\langle I\rangle$ and modulation in$\operatorname{dex} m$ are within $10 \%$ over these three cases. From this second test we conclude, somewhat conservatively, that $\Delta r=s_{0} / 4$ is necessary for the convergence of the computation, i.e. the Kolmogorov spectrum $P_{3 N}$ must include $q_{\max }=\frac{1}{2 s_{0} / 4}$.
As a third test, we study the impact of the spatial resolution $\mathrm{d} x_{\mathrm{s}}$ used to read the phase screen file into Eq. (5). Although we have just concluded that $\Delta r=s_{0} / 4$ is necessary to include enough high frequencies, the phases can possibly be read with a lower resolution. Using the phase screen file constructed with $C_{n}^{2}=10^{-3}, z=3.6 \mathrm{kpc}, N=2^{17}$ and $\Delta r=s_{0} / 4$, we test the three cases: $\mathrm{d} x_{\mathrm{s}}=s_{0}$ in reading every other four phases, $\mathrm{d} x_{\mathrm{s}}=s_{0} / 2$ in reading every other two phases and $\mathrm{d} x_{\mathrm{s}}=s_{0} / 4$ in reading every phase. The integration surface is fixed to $S=\left(4 r_{\mathrm{S}}\right)^{2}$ in this test. Figure B.4 shows the three dynamic spectra corresponding to these resolutions. As the figure shows, they have the same morphology and their averaged intensities changed by only $6 \%$. We extended the resolution further to $\mathrm{d} x_{\mathrm{s}}=2 s_{0}$ and found then that the morphology of the dynamic spectrum becomes abruptly very dissimilar (not shown in Fig. B.4) meaning it had not yet converged. We also computed the intensity series for significant fractions of the screen and report $\langle I\rangle$ and $m$ in Table B.3. From this third test, we conclude that $\mathrm{d} x_{\mathrm{s}}=s_{0}$ is sufficient.

Finally, we note that the resulting modulation index $m(\sim 0.5)$ is larger than the theoretical value $m_{\mathrm{r}}=0.32$ derived for purely refractive scintillation by Gupta et al. (1993) $\left(m_{\mathrm{r}}=\right.$ $\left.1.08\left(\frac{\Delta v_{\mathrm{dc}}}{v}\right)^{1 / 6}\right)$. This is because the size of the dynamic spectra simulated $(70 \mathrm{~min} \times 8.8 \mathrm{MHz})$ is not much larger than the 
Dynamical spectrum $\left(\delta \phi=0^{\circ}\right)$
averaged intensity $=1.68016$

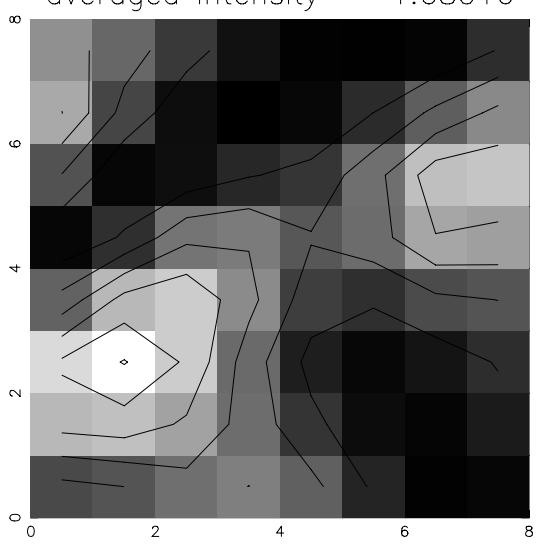

Dynamical spectrum $\left(\delta \phi=32^{\circ}\right)$ averaged intensity $=1.67927$

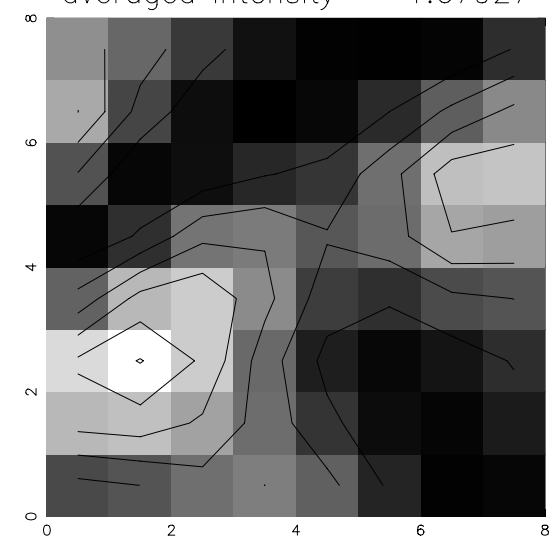

Dynamical spectrum $\left(\delta \phi=10^{\circ}\right)$

averaged intensity $=1.68030$

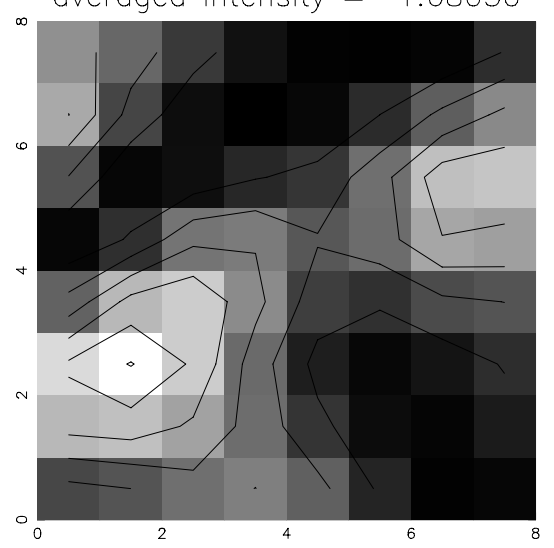

Dynamical spectrum $\left(\delta \phi=57^{\circ}\right)$
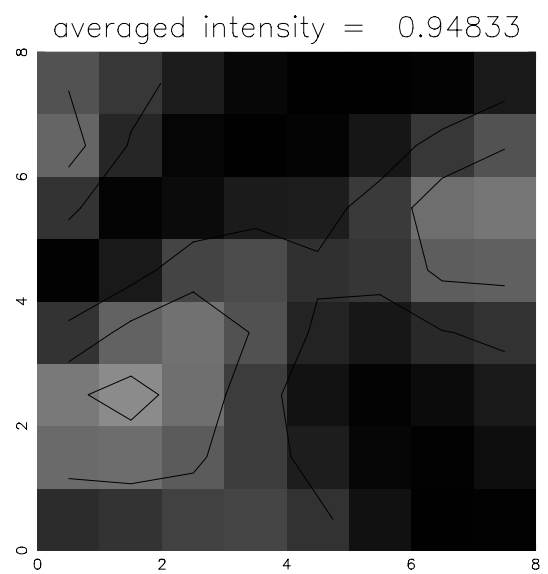

Dynamical spectrum $\left(\delta \phi=18^{\circ}\right)$

averaged intensity $=1.68037$

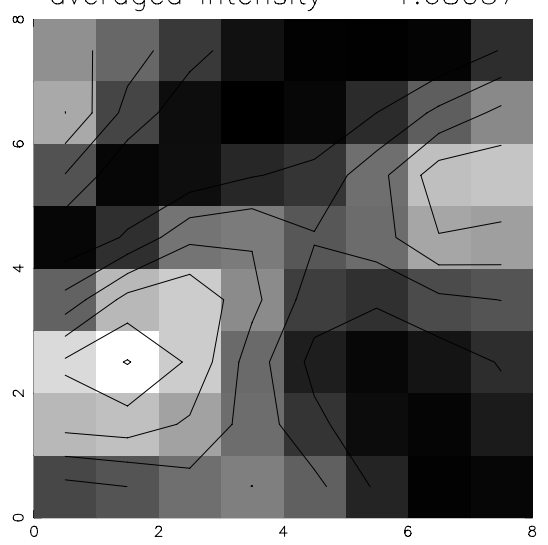

Dynamical spectrum $\left(\delta \phi=101^{\circ}\right)$

averaged intensity $=0.24807$

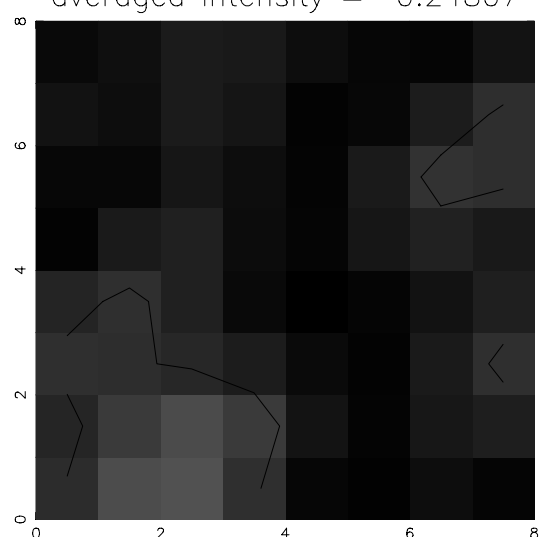

Fig. B.3. Test on parameter $\Delta r$ : dynamic spectra computed at $1.41 \mathrm{GHz}$ at the same telescope location and with the same integration surface size $S=\left(4 r_{\mathrm{S}}\right)^{2}$ but with different grid step $\Delta r$ for the phase screen $\phi_{K}(m, n)$ in Eq. (4). The limits of the Kolmogorov spectrum are directly related to $\Delta r$ and $N$ as $q_{\min }=\frac{1}{N \Delta r}$ and $q_{\max }=\frac{1}{2 \Delta r}$. A full test would require to construct effectively several Kolmogorov phase screens in decreasing $\Delta r$ to modify $q_{\max }$ and in increasing $N$ to keep the same $q_{\min }$. This is very difficult to implement numerically (see text) and, instead, we have simulated this effect in superimposing a corrugated surface onto the original phase screen. This surface is characterized by an amplitude $\delta \phi$ for some spatial frequency $q$. The idea is to find out which perturbating surface $\delta \phi$ is required to modify significantly either the averaged intensity or the morphology of the resulting dynamic spectrum. Our computation covered the following cases: $\delta \phi=10^{\circ}$ for $q=\frac{1}{2 s_{0} / 8} ; \delta \phi=18^{\circ}$ for $q=\frac{1}{2 s_{0} / 4}$; $\delta \phi=32^{\circ}$ for $q=\frac{1}{2 s_{0} / 2} ; \delta \phi=57^{\circ}$ for $q=\frac{1}{2 s_{0}} ; \delta \phi=101^{\circ}$ for $q=\frac{1}{4 s_{0}}$. These amplitudes $\delta \phi$ are derived from the phase structure function $D_{\phi}(s)$ for the separations $s=1 / 8 s_{0}, s=1 / 4 s_{0}, s=1 / 2 s_{0}, s=1 s_{0}, s=2 s_{0}$, respectively. The first panel of the figure shows the dynamic spectrum computed with the original Kolmogorov screen $\left(\delta \phi=0^{\circ}\right)$ constructed with $C_{n}^{2}=10^{-3}, z=3.6 \mathrm{kpc}, N=2^{17}$ and $\Delta r=s_{0} / 4$. This test shows that $q_{\max }=\frac{1}{2 s_{0} / 2}$, i.e. $\Delta r=s_{0} / 2$, is necessary to include all relevant Fourier components for the computation of dynamic spectra. The gray scale and contours are the same for all panels. Unity corresponds to $1.1 \mathrm{MHz}$ along the frequency axis (vertical) and to 8.8 min along the time axis (horizontal) with the screen speed $V=50 \mathrm{~km} \mathrm{~s}^{-1}$.

Table B.3. Sensitivity of the averaged intensity and modulation index to $\mathrm{d} x_{\mathrm{s}}{ }^{a}$.

\begin{tabular}{lcccc}
\hline \hline Resolution $\mathrm{d} x_{\mathrm{s}}$ & $\begin{array}{c}\text { Sampling } \\
\text { of dyn. sp. }\end{array}$ & $\begin{array}{c}\text { Nb of } \\
\text { intensities }\end{array}$ & $\langle I\rangle$ & Index $m$ \\
\hline $1 s_{0}$ & $8 \times 8$ & 69 & 1.57 & 0.54 \\
$s_{0} / 2$ & $16 \times 16$ & 31 & 1.87 & 0.49 \\
$s_{0} / 4$ & $32 \times 32$ & 18 & 1.98 & 0.49 \\
\hline
\end{tabular}

a $S=\left(4 r_{\mathrm{S}}\right)^{2}$ and $q_{\max }=\frac{1}{2 s_{0} / 4}$ kept fixed.

diffractive patches $t_{\mathrm{d}} \times \Delta v_{\mathrm{dc}}=8.8 \mathrm{~min} \times 1.1 \mathrm{MHz}\left(t_{\mathrm{d}}=s_{0} / \mathrm{V}\right.$ and $\left.\Delta v_{\mathrm{dc}}=1 /\left(2 \pi \tau_{\mathrm{S}}\right)\right)$. This size $(70 \mathrm{~min} \times 8.8 \mathrm{MHz})$ we used to average out diffractive scintillation is not large enough and so we have a blend of both refraction and diffraction into $m$. We note also that the normalized intensity $\langle I\rangle$ is $\sim 1.5$ rather than unity but this is a statistical fluctuation. For instance, we found $\langle I\rangle=0.77$ and $m=0.51$ for scintillation simulated along a full $4 r_{\mathrm{S}}$ wide track in another part of our large phase screen. In Fig. B.5, we provide an example of 11 dynamic spectra 2.5 days apart and their summed autocorrelation function. The visibility at $\sigma=s_{0}$ $(\sim 60 \%)$ in $\left\langle E(s) E^{*}(s+\sigma)\right\rangle=\exp \left[-0.5\left(\sigma / s_{0}\right)^{\alpha}\right]$ (Rickett 1988) is closely delineated by the contours $65 \%$ in the $2 \mathrm{D}$ autorrelation function of Fig. B.5. The half widths of this function are $\sim 4 \mathrm{~min}$ and $\sim 0.5 \mathrm{MHz}$ and are consistent with the theoretical values $t_{\mathrm{d}}=8.8 \mathrm{~min}$ and $\Delta v_{\mathrm{dc}}=1.1 \mathrm{MHz}$. 

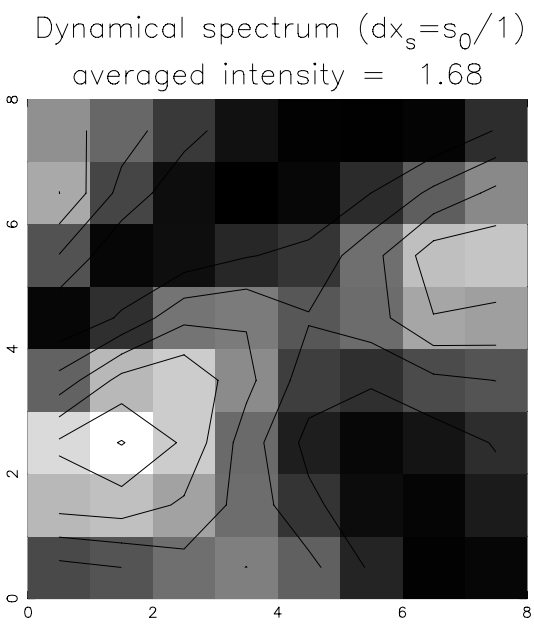

Dynamical spectrum $\left(\mathrm{dx}_{\mathrm{s}}=\mathrm{s}_{0} / 2\right)$ averaged intensity $=1.75$

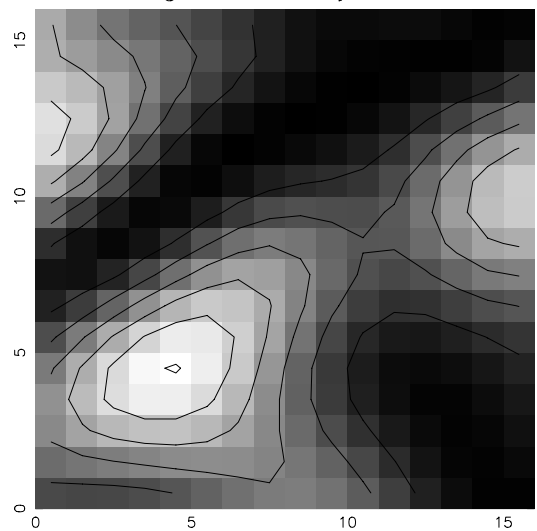

Dynamical spectrum $\left(\mathrm{dx}_{\mathrm{s}}=\mathrm{s}_{0} / 4\right)$ averaged intensity $=1.78$

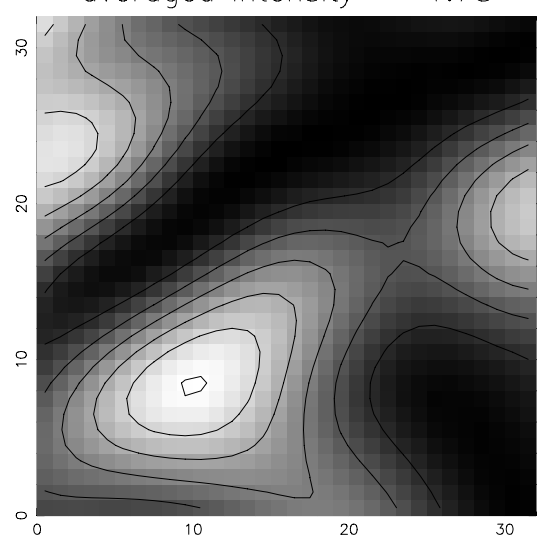

Fig. B.4. Test on the parameter $\mathrm{d} x_{\mathrm{s}}$ : dynamic spectrum computed at $1.41 \mathrm{GHz}$ at the same telescope location and with the same integration surface $S=\left(4 r_{\mathrm{S}}\right)^{2}$ but with different spatial resolution $\mathrm{d} x_{\mathrm{s}}$ in reading the phase screen file. The phase screen $\phi_{K}(m, n)$ has been constructed with $C_{n}^{2}=10^{-3}$, $z=3.6 \mathrm{kpc}, N=2^{17}$ and $\Delta r=s_{0} / 4$. Only every other 4 pixels are read from the phase file into Eq. (5) in the case d $x_{\mathrm{s}}=s_{0}$; only every other 2 pixels for $\mathrm{d} x_{\mathrm{s}}=s_{0} / 2$; every pixel for $\mathrm{d} x_{\mathrm{s}}=s_{0} / 4$ matching $\Delta r=s_{0} / 4$ in that case. The size of the dynamic spectrum is the same for the three panels, $70 \mathrm{~min} \times 8.8 \mathrm{MHz}$, sampled over $8 \times 8,16 \times 16$ and $32 \times 32$ pixels, respectively, and unity is correspondingly $1.1 \mathrm{MHz}, 0.55 \mathrm{MHz}, 0.275 \mathrm{MHz}$ along the frequency axis (vertical) and $8.8 \mathrm{~min}, 4.4 \mathrm{~min}, 2.2 \mathrm{~min}$ along the time axis (horizontal) with the screen speed $V=50 \mathrm{~km} \mathrm{~s}{ }^{-1}$. This test shows that the spatial resolution $\mathrm{d} x_{\mathrm{s}}=s_{0}$ is sufficient to make the computation of dynamic spectra and averaged intensities $\left\langle i\left(\boldsymbol{x}^{\prime}, \lambda\right)\right\rangle$ convergent.
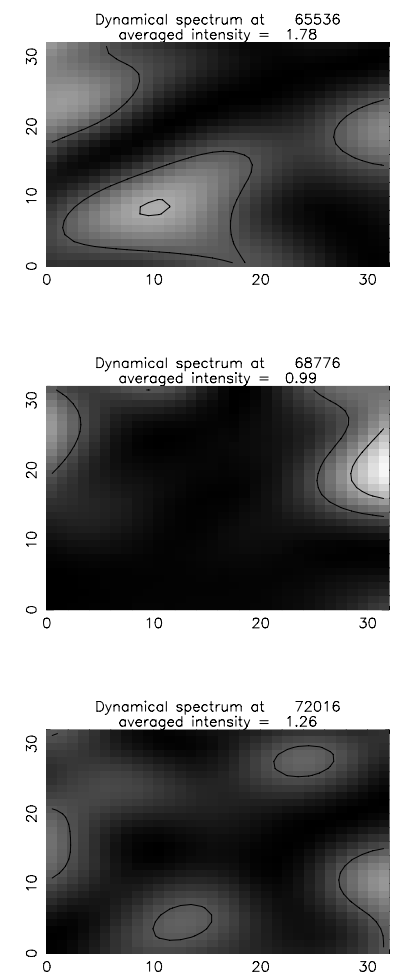

Dynamical spectrum at 66346

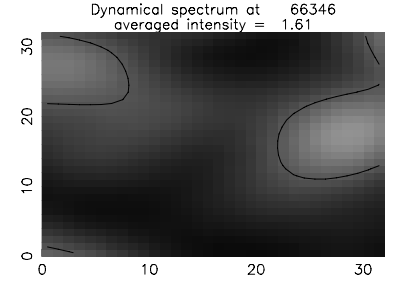

Dynamical spectrum ot 69586

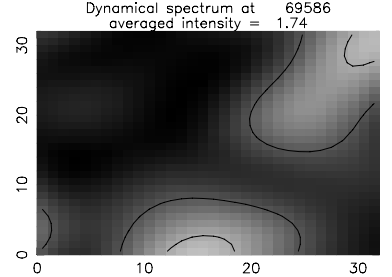

Dynamical spectrum at 72826

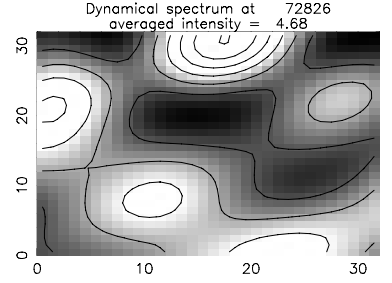

Dynamical spectrum at 67156

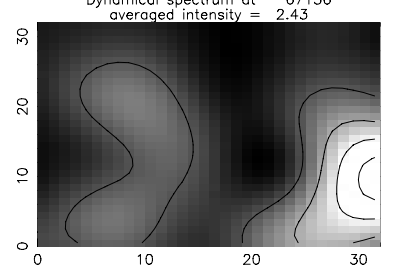

Dynamical spectrum at 70396

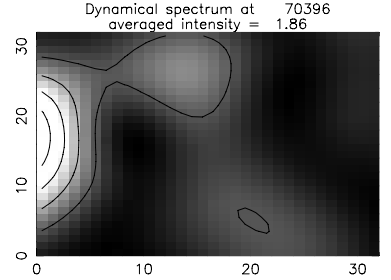

Dynamical spectrum at 73636

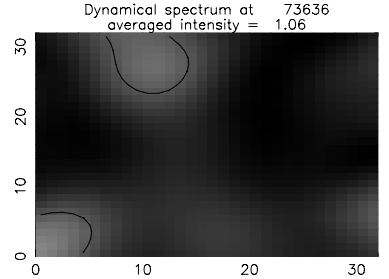

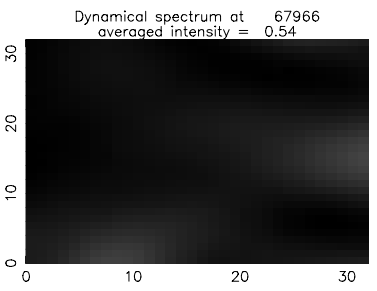

Dynamical spectrum at 71206

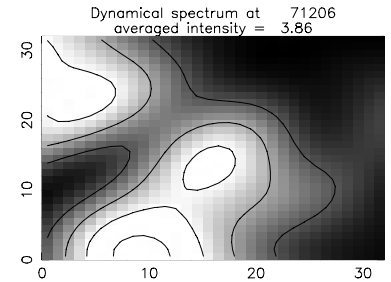

Summed outocrelation fct

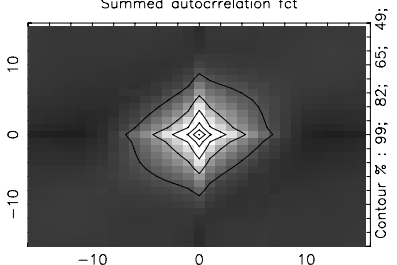

Fig. B.5. Sample of simulated dynamic spectra at $1.4 \mathrm{GHz}$ and their summed autocorrelation function (last panel). All the dynamic spectra are plotted with the same intensity scale, they are 1.25 days apart with the adopted screen speed $V=50 \mathrm{~km} \mathrm{~s}^{-1}$. They show a high variability in morphology and averaged intensity as expected for pulsars. The structure in these dynamic spectra is similar to speckles seen in images at optical wavelengths. Their summed autocorrelation provides a mean to measure the diffactive time scale and decorrelation bandwidth. We found they match the theoretical values (see text). Unity corresponds to $0.275 \mathrm{MHz}$ along the frequency axis (vertical) and to $2.2 \mathrm{~min}$ along the time axis (horizontal) for both the dynamic spectra and the summed autocorrelation function. This simulation is done with $C_{n}^{2}=10^{-3}, z=3.6 \mathrm{kpc}$, $\mathrm{d} x_{\mathrm{s}}=\Delta r=s_{0} / 4, N=2^{17}$ and $S=\left(4 r_{\mathrm{S}}\right)^{2}$.

In Fig. B.6, we compute the normalized autocorrelation function $(a c f(\tau) / a c f(0))$ of the time series of the intensity shown in Fig. B. 1 for the cases $S=\left(4 r_{\mathrm{S}}\right)^{2}$. This function shows the slow decline, $\sim 10$ days at half-maximum of $\operatorname{ac} f(1) / \operatorname{ac} f(0)$, expected because of the correlation induced by the long refractive scales of the phase screen. This is consistent with the 


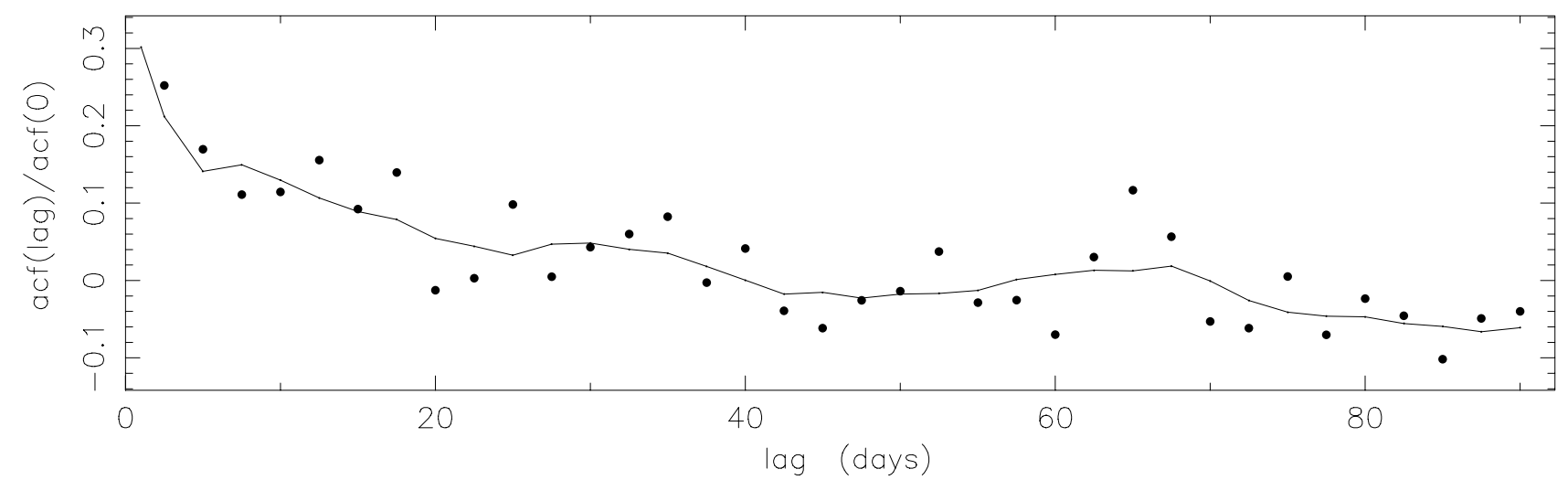

Fig. B.6. Autocorrelation function of the time series of the intensity computed with $S=\left(4 r_{\mathrm{S}}\right)^{2}$ and shown as filled triangles in Fig. B.1. The refraction time scale of $\sim 10$ days at half-maximum of this function is consistent with the theoretical value of 15 days.

theoretical refractive time scale $\tau_{\mathrm{R}}=L \theta_{\mathrm{S}} / V$ (Rickett 1988) of 15 days with $L=1.8 \mathrm{kpc}, V=50 \mathrm{~km} \mathrm{~s}^{-1}$ and the scattering angle $\theta_{\mathrm{S}}=\frac{\lambda}{2 \pi s_{0}}=1.25 \times 10^{-9} \mathrm{Rd}$, with $\lambda=0.21 \mathrm{~m}$ and $s_{0}=2.66 \times 10^{7} \mathrm{~m}$.

\section{References}

Armstrong, J. W., Rickett, B. J., \& Spangler, S. R. 1995, ApJ, 443, 209 Blandford, R., \& Narayan, R. 1985, MNRAS, 213, 591

Born, M., \& Wolf, E. 1999, Principles of Optics, 7th edn. (Cambridge University Press)

Cognard, I., Bourgois, G., Lestrade, J.-F., et al. 1993, Nature, 366, 320

Coles, W. A., Filice, J. P., Frehlich, R. G., \& Yadlowsky, M. 1995, Appl. Opt., 34, 2089

Cordes, J. M., Weisberg, J. M., \& Boriakoff, V. 1985, ApJ, 288, 221

Cordes, J. M., Pidwervetsky, A., \& Lovelace, R. V. E. 1986, ApJ, 310, 737

Deshpande, A. 2000, MNRAS, 317, 199

Fiedler, R. L., Dennison, B., Johnston, K. J., \& Hewish, A. 1987, Nature, 326, 675

Fiedler, R. L., Dennison, B., Johnston, K. J., Waltman, E. B., \& Simon, R. S. 1994, ApJ, 430, 581

Goodman, J. W. 1968, Introduction to Fourier Optics (New York: McGraw-Hill)

Gupta, Rickett, B. J., \& Coles, W. A. 1993, ApJ, 403, 183

Gupta, Rickett, B. J., \& Lyne, A. 1994, MNRAS, 269, 1035

Gwinn, C. R., Britton, M. C., Reynolds, J. E., et al. 1998, ApJ, 505, 928

Hamidouche, M. 2003, Ph.D. Thesis, LPCE-CNRS, University of Orleans, Paris Observatory
Hamidouche, M., Lestrade, J.-F., \& Cognard, I. 2002, in Proceedings Scientific Highlights 2002 of the Société Française d'Astronomie et d'Astrophysique, Paris, June 2002, ed. F. Combes, \& D. Barret, F. (EDP Sciences), 29

Hill, A. S., Stinebring, D. R., Asplund, C. T., et al. 2005, ApJ, 619, L171

Johnston, S., Nicastro, L., \& Koribalski, B. 1998, MNRAS, 297, 108

Lazio, T. J. W., Waltman, E. B., Ghigo, F. D., et al. 2001, ApJS, 136, 265

Lestrade, J.-F., Rickett, B. J., \& Cognard, I. 1998, A\&A, 334, 1068

Lovelace, R. V. E. 1970, Ph.D. Thesis, Cornell University

Lovelace, R. V. E., Salpeter, E. E., Sharp, L. E., \& Harris, D. E. 1970, ApJ, 159, 1047

Maitia, V., Lestrade, J.-F., \& Cognard, I. 2003, ApJ, 582, 2, 972

Narayan, R. 1992, Phil. Trans. R. Soc. Lond. A, 341, 151

Papoulis, A. 1965, Probability, Random Variable and Stochastic Process (McGraw-Hill)

Rice, S. O. 1944, Mathematical Analysis of Random Noise, Bell System Tech. J., 23, 282

Rickett, B. J. 1977, ARA\&A, 15, 479

Rickett, B. J. 1988, in Radio wave scattering in the interstellar medium, Proceedings of the AIP Conference, San Diego, CA, Jan. 18-19, 1988, New York, American Institute of Physics, 2

Rickett, B. J. 1990, ARA\&A, 28, 561

Romani, R. W., Blandford, R. D., \& Cordes, J. M. 1987, Nature, 328, 324

Ryba, M. F. 1991, High Precision Timing of Millisecond Pulsars, Ph.D. Thesis Princeton University

Salpeter, E. E. 1967, ApJ, 147, 433

Stinebring, D. R., Smirnova, T. V., Hankins, H., et al. 2000, ApJ, 539, 300

Thompson, A. R., Moran, J. M., \& Swenson, G. W. 1986, Interferometry and Synthesis in Radioastronomy, 1rst edn. (Wiley-Interscience) 